\title{
Bayesian Filtering With Random Finite Set Observations
}

\author{
Ba-Tuong Vo, Ba-Ngu Vo, and Antonio Cantoni, Fellow, IEEE
}

\begin{abstract}
This paper presents a novel and mathematically rigorous Bayes' recursion for tracking a target that generates multiple measurements with state dependent sensor field of view and clutter. Our Bayesian formulation is mathematically well-founded due to our use of a consistent likelihood function derived from random finite set theory. It is established that under certain assumptions, the proposed Bayes' recursion reduces to the cardinalized probability hypothesis density (CPHD) recursion for a single target. A particle implementation of the proposed recursion is given. Under linear Gaussian and constant sensor field of view assumptions, an exact closed-form solution to the proposed recursion is derived, and efficient implementations are given. Extensions of the closed-form recursion to accommodate mild nonlinearities are also given using linearization and unscented transforms.
\end{abstract}

Index Terms-Bayesian filtering, CPHD filter, Gaussian sum filter, Kalman filter, particle filter, PHD filter, point processes, random finite sets, target tracking.

\section{INTRODUCTION}

$\mathbf{T}$ HE objective of target tracking is to estimate the state of the target from measurement sets collected by a sensor at each time step. This is a challenging problem since the target can generate multiple measurements which are not always detected by the sensor, and the sensor receives a set of spurious measurements (clutter) not generated by the target. Many existing techniques for handling this problem rest on the simplifying assumptions that the target generates at most one measurement and that the sensor field of view is constant. Such assumptions are not realistic, for example, in extended object tracking or tracking in the presence of electronic counter measures, which are increasingly becoming important due to high-resolution capabilities of modern sensors. Nonetheless, these assumptions have formed the basis of a plethora of works e.g., the multiple hypothesis tracker (MHT) [1], [2], the probabilistic data association (PDA) filter [3], the Gaussian mixture filter [4], the integrated PDA (IPDA) filter [5], and their variants. However, such techniques are not easily adapted to accommodate multiple measurements

Manuscript received February 28, 2007; revised July 31, 2007. The associate editor coordinating the review of this manuscript and approving it for publication was Dr. Subhrakanti Dey.

B.-T. Vo and A. Cantoni are with the Western Australian Telecommunications Research Institute, University of Western Australia, Crawley, WA 6009, Australia (e-mail: vob@watri.org.au; cantoni@watri.org.au).

B.-N. Vo is with the Electrical Engineering Department, University of Melbourne, Parkville, VIC 3010, Australia (e-mail: bv@ee.unimelb.edu.au).

Color versions of one or more of the figures in this paper are available online at http://ieeexplore.ieee.org.

Digital Object Identifier 10.1109/TSP.2007.908968 generated by the target and state dependent field of view. Moreover, it is not clear how such techniques are mathematically consistent with the Bayesian paradigm.

Tracking in the presence of detection uncertainty, data association uncertainty and clutter can be elegantly cast in the Bayesian filtering paradigm using random finite set (RFS) modeling. While this idea has been advocated by various researchers [6]-[8], Mahler's random finite set approach (coined as finite set statistics or FISST) is the first systematic Bayesian framework for the study of multisensor multitarget tracking [9]-[11]. Exciting advances and developments in the random finite set approach have attracted substantial interest in recent years, especially moment approximations for multitarget tracking [10]-[15]. In the single-target realm, however, the RFS approach has not been utilized to further advance single-target tracking techniques, though connections with existing techniques such as PDA and IPDA have been addressed [16].

To the best of the authors' knowledge, this paper is the first to use the RFS formalism to solve the problem of tracking a target that can generate multiple measurements, in the presence of detection uncertainty and clutter. In our Bayesian filtering formulation, the collection of observations at any time is treated as a set-valued observation which encapsulates the underlying models of multiple target-generated measurements, state dependent sensor field of view, and clutter. Central to the Bayes' recursion is the concept of a probability density. Since the observation space is now the space of finite sets, the usual Euclidean notion of a density is not suitable. An elegant and rigorous notion of a probability density needed for the Bayes' recursion is provided by RFS or point process theory [9], [10], [12], [13].

The contributions of this paper are as follows.

- A novel and mathematically rigorous Bayes' recursion together with a particle implementation that accommodates multiple measurements generated by the target, state dependent field of view and clutter using RFS theory,

- A closed-form solution to the proposed recursion for linear Gaussian single-target models with constant sensor field of view, and extensions to accommodate mild nonlinearities using linearization and unscented transforms.

- Under certain assumptions the proposed Bayes' recursion is shown to reduce to Mahler's cardinalized probability hypothesis density (CPHD) recursion [14] restricted to a single target.

- Our approach is compared with conventional techniques and is shown to be significantly better in terms of track loss and localization performance.

In contrast to the traditional approaches [1]-[5], our proposed recursion formally accommodates multiple measurements generated by the target, detection uncertainty and clutter, thereby 
providing a formal mechanism for handling effects such as electronic counter measures and multipath reflections. Indeed, assuming no clutter and that the target generates exactly one measurement, the proposed recursion reduces to the usual Bayes' recursion and the particle filter implementation reduces to the standard particle filter. Under additional linear Gaussian assumptions, our closed-form recursion reduces to the celebrated Kalman filter (hence our extensions via linearization and unscented transforms reduce to the extended and unscented Kalman filters, respectively). In the case of a linear Gaussian model with at most one target-generated measurement, constant field of view, and uniform clutter, the proposed closed-form recursion reduces to the Gaussian mixture filter given in [4]. Moreover, if at each time step, gating is performed and the Gaussian mixture posterior density is collapsed to a single Gaussian component, then the proposed recursion reduces to the PDA filter [3].

We have announced some of the results of the current work in the conference paper [17].

The paper is structured as follows. Section II presents background information on Bayesian filtering and random finite sets. Section III then formulates the single-target tracking problem in the Bayes framework that accommodates multiple measurements generated by the target, state dependent sensor field of view, and clutter; this section also establishes the connection between the proposed recursion and Mahler's CPHD recursion restricted to a single target. A particle implementation of the proposed recursion is presented in Section IV along with a nonlinear demonstration and numerical studies. An exact closed-form solution to the proposed recursion is derived for linear Gaussian single-target models in Section $\mathrm{V}$ along with demonstrations and numerical studies. Extensions of the closed-form recursion to accommodate nonlinear Gaussian models are described in Section VI. Concluding remarks are given in Section VII.

\section{BACKGROUND}

\section{A. The Bayes' Recursion}

In the classical Bayes' filter [18], [19] the hidden state $x_{k}$ is assumed to follow a first-order Markov process on the state space $\mathcal{X} \subseteq \mathbb{R}^{n_{x}}$ according to a transition density $f_{k \mid k-1}\left(x_{k} \mid x_{k-1}\right)$, which is the probability density that the target with state $x_{k-1}$ at time $k-1$ moves to state $x_{k}$ at time $k$. The observation $z_{k} \in \mathcal{Z} \subseteq \mathbb{R}^{n_{z}}$ is assumed conditionally independent given the states $x_{k}$ and is characterized by a likelihood $g_{k}\left(z_{k} \mid x_{k}\right)$, which is the probability density that, at time $k$, the target with state $x_{k}$ produces a measurement $z_{k}$. Under these assumptions, the classical Bayes' recursion propagates the posterior density $p_{k}$ in time according to

$$
\begin{aligned}
p_{k \mid k-1}\left(x_{k} \mid z_{1: k-1}\right) & =\int f_{k \mid k-1}\left(x_{k} \mid x\right) p_{k-1}\left(x \mid z_{1: k-1}\right) d x \\
p_{k}\left(x_{k} \mid z_{1: k}\right) & =\frac{g_{k}\left(z_{k} \mid x_{k}\right) p_{k \mid k-1}\left(x_{k} \mid z_{1: k-1}\right)}{\int g_{k}\left(z_{k} \mid x\right) p_{k \mid k-1}\left(x \mid z_{1: k-1}\right) d x}
\end{aligned}
$$

where $z_{1: k}=\left[z_{1}, \ldots, z_{k}\right]$. All inference on the target state at time $k$ is derived from the posterior density $p_{k}$. Common estimators for the target state are the expected a posteriori (EAP) and maximum a posteriori (MAP) estimators.

The Bayes' recursion (1), (2) is formulated for single-target single-measurement systems. In practice due to multipath reflections, electronic counter measures, etc., the target may generate multiple measurements, in addition to spurious measurements not generated by the target. Note that at any given time step, the order of appearance of measurements received by sensor has no physical significance. Hence, at time $k$ the sensor effectively receives an unordered set of measurements denoted by $Z_{k}$, and the observation space is now the space of finite subsets of $\mathcal{Z}$, denoted by $\mathcal{F}(\mathcal{Z})$. Consequently, the Bayes' update (2) is not directly applicable.

To accommodate set-valued measurements, we require a mathematically consistent generalization of the likelihood $g_{k}\left(z_{k} \mid x_{k}\right)$ to the set-valued case. In other words, we need a mathematically rigorous notion of the probability density of the set $Z_{k}$ given $x_{k}$. However, the notion of such densities is not straightforward because the space $\mathcal{F}(\mathcal{Z})$ does not inherit the usual Euclidean notions of volume and integration on $\mathcal{Z}$. We review in the next subsection how RFS theory or point process theory provides rigorous notions of volume and integration on $\mathcal{F}(\mathcal{Z})$ needed to define a mathematically consistent likelihood.

\section{B. Random Finite Sets}

We describe in this subsection the bare minimum background on RFS theory needed to develop the results in this paper. For a classical treatment of the mathematical theory of RFSs (or point processes), the reader is referred to [20] and [21], while a comprehensive treatment of multitarget tracking using RFSs can be found in [9]-[11].

Let $(\Omega, \sigma(\Omega), P)$ be a probability space, where $\Omega$ is the sample space, $\sigma(\Omega)$ is a $\sigma$-algebra on $\Omega$, and $P$ is a probability measure on $\sigma(\Omega)$. A random finite set $Z$ on a complete separable metric space $\mathcal{Z}$ (e.g., $\mathcal{Z}=\mathbb{R}^{n_{z}}$ ) is defined as a measurable mapping

$$
\Sigma: \Omega \rightarrow \mathcal{F}(\mathcal{Z})
$$

with respect to the Borel sets of $\mathcal{F}(\mathcal{Z})$ [20]-[22]. ${ }^{1}$ The probability distribution of the RFS $\Sigma$ is given in terms of the probability measure $P$ by

$$
\operatorname{Pr}(\Sigma \in \mathcal{T})=P(\{\omega \in \Omega: \Sigma(\omega) \in \mathcal{T}\})
$$

where $\mathcal{T}$ is any Borel subset of $\mathcal{F}(\mathcal{Z})$. The probability distribution of the RFS $\Sigma$ can be equivalently characterized by a discrete probability distribution and a family of joint probability distributions. The discrete distribution characterizes the cardinality (the number of elements) of the RFS, whilst for a given cardinality, an appropriate distribution characterizes the joint distribution of the elements of the RFS [20]-[22].

The probability density $p_{\Sigma}$ of $\Sigma$ is given by the Radon-Nikodým derivative of its probability distribution with respect to an appropriate dominating measure $\mu$. i.e.,

\footnotetext{
${ }^{1}$ Technically, $\mathcal{F}(\mathcal{Z})$ is embedded in the (complete separable metric) space of
} counting measures on $\mathcal{Z}$, and inherits the Borel sets of this space. 
$\operatorname{Pr}(\Sigma \in \mathcal{T})=\int_{\mathcal{T}} p_{\Sigma}(Z) \mu(d Z)$. The conventional choice of dominating measure is [23]

$$
\mu(\mathcal{T})=\sum_{r=0}^{\infty} \lambda^{r}\left(\chi^{-1}(\mathcal{T}) \cap \mathcal{Z}^{r}\right) / r !
$$

where $\lambda^{r}$ is the $r$ th product (unitless) Lebesque measure, $\chi$ is a mapping of vectors to sets defined by $\chi\left(\left[z_{1}, \ldots, z_{r}\right]^{T}\right)=\left\{z_{i}\right.$ : $i=1, \ldots, r\}$, and $\mathcal{Z}^{r}$ is the $r$ th Cartesian product of $\mathcal{Z}$ with the convention $\mathcal{Z}^{0}=\{\emptyset\}$. The integral of a measurable function $f: \mathcal{F}(\mathcal{Z}) \rightarrow \mathbb{R}$ with respect to $\mu$ is defined as follows:

$$
\begin{aligned}
& \int_{\mathcal{T}} f(Z) \mu(d Z)=\sum_{r=0}^{\infty} \frac{1}{r !} \int_{\chi^{-1}(\mathcal{T}) \cap \mathcal{Z}^{r}} f\left.\left\{z_{1}, \ldots, z_{r}\right\}\right) \\
& \times \lambda^{r}\left(d z_{1} \ldots d z_{r}\right) .
\end{aligned}
$$

The first-order moment of a random finite set $\Sigma$ on $\mathcal{Z}$, also called the intensity function, is a non-negative function $v_{\Sigma}$ on $\mathcal{Z}$ with the property that for any closed subset $S \subseteq \mathcal{Z}$

$$
\mathbb{E}[|\Sigma \cap S|]=\int_{S} v_{\Sigma}(x) d x
$$

where $|\Sigma|$ denotes the cardinality of $\Sigma$. In other words, for a given point $x$, the intensity $v_{\Sigma}(x)$ is the density of expected number of targets per unit volume at $x$.

An important class of RFSs are the Poisson RFSs (or Poisson point processes) [20], which are completely characterized by their intensity functions. The cardinality of a Poisson RFS $\Sigma$ is Poisson distributed with mean $N_{\Sigma}=\int v_{\Sigma}(x) d x$, and for a given cardinality the elements of $\Sigma$ are each independent and identically distributed (i.i.d.) with probability density $v_{\Sigma} / N_{\Sigma}$. More generally, an RFS whose elements are i.i.d. according to $v_{\Sigma} / N_{\Sigma}$, but has arbitrary cardinality distribution is called an i.i.d. cluster process [20].

For simplicity in notation, we shall use the same symbol for an RFS and its realizations hereon.

\section{RFS SINGLE-TARGET BAYES' RECURSION}

The classical Bayes' filter was formulated for the case where the target generates exactly one measurement and there is no clutter. Hence, in the classical Bayes' filter, the measurement is vector-valued and modeled as a random variable given by a likelihood function defined on $\mathcal{Z}$. As previously argued, in the presence of multiple measurements generated by the target, detection uncertainty and clutter, the measurement is set-valued. In this section, we describe a RFS measurement model and derive the corresponding likelihood function on $\mathcal{F}(\mathcal{Z})$.

\section{A. RFS Measurement Model}

The collection of measurements obtained at time $k$ is represented as a finite subset $Z_{k}$ of the original observation space $\mathcal{Z} \subseteq \mathbb{R}^{n_{z}}$. More concisely, if $M(k)$ observations $z_{k, 1}, \ldots, z_{k, M(k)} \in \mathcal{Z}$ are received at time $k$, then

$$
Z_{k}=\left\{z_{k, 1}, \ldots, z_{k, M(k)}\right\} \in \mathcal{F}(\mathcal{Z}) .
$$

Suppose at time $k$ that the target is in state $x_{k}$. The measurement process is given by the RFS measurement equation

$$
Z_{k}=\Theta_{k}\left(x_{k}\right) \cup E_{k}\left(x_{k}\right) \cup W_{k}
$$

where $\Theta_{k}\left(x_{k}\right)$ is the RFS of the primary target-generated measurement, $E_{k}\left(x_{k}\right)$ is the RFS of extraneous target-generated measurements, and $W_{k}$ is the RFS of clutter. For example, $\Theta_{k}\left(x_{k}\right)$ may represent a single direct path measurement, $E_{k}\left(x_{k}\right)$ may represent measurements generated by multipath effects or counter measures, and $W_{k}$ may represent state independent spurious measurements. It is assumed that conditional on $x_{k}, \Theta_{k}\left(x_{k}\right), E_{k}\left(x_{k}\right)$ and $W_{k}$ are independent RFSs.

We model $\Theta_{k}\left(x_{k}\right)$ as a binary RFS

$$
\Theta_{k}\left(x_{k}\right)= \begin{cases}\emptyset & \text { with probability } 1-p_{D, k}\left(x_{k}\right) \\ \left\{z_{k}^{*}\right\} & \text { with probability density } p_{D, k}\left(x_{k}\right) g_{k}\left(z_{k}^{*} \mid x_{k}\right)\end{cases}
$$

where $p_{D, k}(\cdot)$ is the probability of detection for the primary measurement, and $g_{k}(\cdot \mid \cdot)$ is the likelihood for the primary measurement. Hence, the probability of not obtaining the primary measurement from a state $x_{k}$ is $1-p_{D, k}\left(x_{k}\right)$, and conversely, given that there is a primary measurement the probability density of obtaining the primary measurement $z_{k}^{*}$ from a state $x_{k}$ is $g_{k}\left(z_{k}^{*} \mid x_{k}\right)$.

We model $E_{k}\left(x_{k}\right)$ and $W_{k}$ in (8) as Poisson RFSs with intensities $v_{E, k}\left(\cdot \mid x_{k}\right)$ and $v_{W, k}(\cdot)$, respectively. For convenience we group these RFSs together as

$$
K_{k}\left(x_{k}\right)=E_{k}\left(x_{k}\right) \cup W_{k} .
$$

Since $K_{k}\left(x_{k}\right)$ is a union of statistically independent Poisson RFSs, it is also a Poisson RFS with intensity

$$
v_{K, k}\left(z_{k} \mid x_{k}\right)=v_{W, k}\left(z_{k}\right)+v_{E, k}\left(z_{k} \mid x_{k}\right) .
$$

The cardinality distribution $\rho_{K, k}\left(\cdot \mid x_{k}\right)$ of $K_{k}\left(x_{k}\right)$ is Poisson with mean $\int v_{K, k}\left(z_{k} \mid x_{k}\right) d z_{k}$. Hence, if the target is in state $x_{k}$ at time $k$, the probability of $K_{k}\left(x_{k}\right)$ having exactly $n_{k}$ measurements is $\rho_{K, k}\left(n_{k} \mid x_{k}\right)$, whilst each measurement $z_{k}$ is independent and identically distributed according to the probability density

$$
c_{k}\left(z_{k} \mid x_{k}\right)=v_{K, k}\left(z_{k} \mid x_{k}\right) / \int v_{K, k}\left(z \mid x_{k}\right) d z .
$$

The following proposition establishes the likelihood corresponding to the above RFS measurement model. See Appendix A for the proof.

Proposition 1: If the measurements follow the RFS model in (8), then the probability density that the state $x_{k}$ at time $k$ produces the measurement set $Z_{k}$ is given by

$$
\begin{aligned}
\eta_{k}\left(Z_{k} \mid x_{k}\right)= & K_{o}^{\left|Z_{k}\right|}\left[1-p_{D, k}\left(x_{k}\right)\right] \rho_{K, k}\left(\left|Z_{k}\right| \mid x_{k}\right)\left|Z_{k}\right| ! \\
& \times \prod_{z_{k} \in Z_{k}} c_{k}\left(z_{k} \mid x_{k}\right)+p_{D, k}\left(x_{k}\right) \\
& \times \rho_{K, k}\left(\left|Z_{k}\right|-1 \mid x_{k}\right) \times\left(\left|Z_{k}\right|-1\right) ! \\
& \times \sum_{z_{k}^{*} \in Z_{k}} g_{k}\left(z_{k}^{*} \mid x_{k}\right) \prod_{z_{k} \neq z_{k}^{*}} c_{k}\left(z_{k} \mid x_{k}\right)
\end{aligned}
$$


where $K_{o}$ denotes the unit of volume on $\mathcal{Z}$, in the sense that $\eta_{k}\left(\cdot \mid x_{k}\right)$ is the Radon-Nikodým derivative of the probability distribution of $Z_{k}$ given $x_{k}$ with respect to the dominating measure (6), i.e.,

$$
\operatorname{Pr}\left(Z_{k} \in \mathcal{T} \mid x_{k}\right)=\int_{\mathcal{T}} \eta_{k}\left(Z_{k} \mid x_{k}\right) \mu\left(d Z_{k}\right) .
$$

Remark: The expression in Proposition 1 is a probability density, and is derived from first principles using only measure theoretic probability concepts. A similar expression has been independently derived by Mahler using finite set statistics (FISST) in [11]. ${ }^{2}$ However, Mahler stresses that the FISST derivative is not a Radon-Nikodým derivative [11, p. 716] and hence is not a probability density. We refer the reader to [10] for more details on the relationship between the FISST set derivative and probability density of RFS.

The likelihood (12) has $\left|Z_{k}\right|+1$ terms each of which admits an intuitive interpretation. The first term relates to a missed primary measurement detection, whilst each of the remaining $\left|Z_{k}\right|$ terms relates to a primary measurement detection. To explain the first term, notice that when there is a missed primary measurement detection, $Z_{k}=K_{k}\left(x_{k}\right)$. Hence, the likelihood of $Z_{k}$ comprises the following: $1-p_{D, k}\left(x_{k}\right)$, the probability of a missed primary measurement detection; $\rho_{K, k}\left(\left|Z_{k}\right| \mid x_{k}\right)$, the probability that $K_{k}\left(x_{k}\right)$ has exactly $\left|Z_{k}\right|$ measurements; $\prod_{z_{k} \in Z_{k}} c_{k}\left(z_{k} \mid x_{k}\right)$, the joint density of the measurements; and a factorial term to account for all possible permutations of $Z_{k}$. To explain each of the $\left|Z_{k}\right|$ remaining terms, notice that when there is a primary measurement detection, $\Theta_{k}\left(x_{k}\right)=\left\{z_{k}^{*}\right\}$ and $K_{k}\left(x_{k}\right)=Z_{k} \backslash\left\{z_{k}^{*}\right\}$. Hence, the likelihood of $Z_{k}$ comprises the following: $p_{D, k}\left(x_{k}\right)$, the probability of a primary measurement detection; $\rho_{K, k}\left(\left|Z_{k}\right|-1 \mid x_{k}\right)$, the probability that $K_{k}\left(x_{k}\right)$ has exactly $\left|Z_{k}\right|-1$ measurements; $g_{k}\left(z_{k}^{*} \mid x_{k}\right) \prod_{z_{k} \neq z_{k}^{*}} c_{k}\left(z_{k} \mid x_{k}\right)$, the joint density of the measurements and a factorial term to account for all possible permutations of $K_{k}\left(x_{k}\right)$.

As a check for consistency, if there is always a primary targetgenerated measurement, no extraneous target-generated measurements and no clutter, i.e., $p_{D, k}\left(x_{k}\right) \equiv 1$ and $\rho_{K, k}\left(n_{k} \mid x_{k}\right)=$ $\delta_{0}\left(n_{k}\right)\left(\delta_{i}(j)=1\right.$ if $i=j$ and zero otherwise), it can be seen that $Z_{k}=\left\{z_{k}^{*}\right\}$ and $\eta_{k}\left(\left\{z_{k}\right\} \mid x_{k}\right)=g_{k}\left(z_{k}^{*} \mid x_{k}\right)$. In other words, the measurement set is always a singleton containing the primary measurement, and the likelihood (12) reduces to the usual single measurement likelihood.

Remark: If $E_{k}\left(x_{k}\right) \equiv \emptyset$ (hence $v_{E, k}(z \mid x)=0$ and $\rho_{E, k}(n \mid x)=\delta_{0}(n)$ ), and $W_{k}$ is an i.i.d. cluster process in (8), (9), then the likelihood (12) still holds. However, if $E_{k}\left(x_{k}\right)$ and $W_{k}$ in (8), (9) are both i.i.d cluster processes, the RFS $K_{k}\left(x_{k}\right)=W_{k} \cup E_{k}\left(x_{k}\right)$ is, in general, no longer an i.i.d. cluster process. Nonetheless if $K_{k}\left(x_{k}\right)$ can be approximated by an i.i.d. cluster process with matching intensity and cardinality distribution

$$
\begin{aligned}
v_{K, k}\left(z_{k} \mid x_{k}\right) & =v_{W, k}\left(z_{k}\right)+v_{E, k}\left(z_{k} \mid x_{k}\right) \\
\rho_{K, k}\left(n_{k} \mid x_{k}\right) & =\left(\rho_{W, k} * \rho_{E, k}\right)\left(n_{k} \mid x_{k}\right)
\end{aligned}
$$

\footnotetext{
${ }^{2}$ The book [11] appeared around the same time that we submitted our preliminary result [17]
}

where $*$ denotes convolution, $\rho_{W, k}(\cdot)$ and $\rho_{E, k}\left(\cdot \mid x_{k}\right)$ are the cardinality distributions of $W_{k}$ and $E_{k}\left(x_{k}\right)$, then the likelihood (12) is still valid.

\section{B. RFS Single-Target Bayes' Recursion}

The Bayes' recursion (1), (2) can be generalized to accommodate multiple measurements generated by the target, detection uncertainty, and clutter, by replacing the standard likelihood $g_{k}\left(z_{k} \mid x_{k}\right)$ with the RFS measurement likelihood $\eta_{k}\left(Z_{k} \mid x_{k}\right)$ in (12). Hence, the posterior density $p_{k}\left(\cdot \mid Z_{1: k}\right)$ can be propagated as follows:

$$
\begin{aligned}
p_{k \mid k-1}\left(x_{k} \mid Z_{1: k-1}\right) & =\int f_{k \mid k-1}\left(x_{k} \mid x\right) p_{k-1}\left(x \mid Z_{1: k-1}\right) d x \\
p_{k}\left(x_{k} \mid Z_{1: k}\right) & =\frac{\eta_{k}\left(Z_{k} \mid x_{k}\right) p_{k \mid k-1}\left(x_{k} \mid Z_{1: k-1}\right)}{\int \eta_{k}\left(Z_{k} \mid x\right) p_{k \mid k-1}\left(x \mid Z_{1: k-1}\right) d x}
\end{aligned}
$$

where $Z_{1: k}=\left[Z_{1}, \ldots, Z_{k}\right]$.

In general, this recursion does not admit an analytic solution. However, the problem can be solved using sequential Monte Carlo techniques as shown in Section IV. Furthermore, a closed-form solution to this recursion can be derived under linear Gaussian assumptions as shown in Section V.

Remark: If there is always a primary target-generated measurement, no extraneous target-generated measurements and no clutter, then $\eta_{k}\left(\left\{z_{k}\right\} \mid x_{k}\right)=g_{k}\left(z_{k} \mid x_{k}\right)$ and the recursion (16), (17) reduces to the classical Bayes' recursion (1), (2).

Remark: The recursion (16), (17) can be easily extended to accommodate multiple sensors. Suppose that there are $S$ mutually independent sensors, i.e., the product of the individual likelihoods for each sensor is the joint likelihood for all sensors. More concisely, if each sensor is modeled by a likelihood $\eta_{k}^{(s)}(\cdot \mid \cdot)$ and receives a measurement set $Z_{k}^{(s)}$ at time $k$ where $s=1, \ldots, S$, then the combined likelihood accounting for all sensors is

$$
\eta_{k}\left(Z_{k}^{(1)}, \ldots, Z_{k}^{(S)} \mid x_{k}\right)=\prod_{s=1}^{S} \eta_{k}^{(s)}\left(Z_{k}^{(s)} \mid x_{k}\right) .
$$

\section{Connection With Mahler's CPHD Filter}

In this section, we show how the proposed RFS single-target Bayes' recursion is related to Mahler's CPHD recursion [14], which is a moment approximation of Mahler's multitarget Bayes' recursion.

The following is a brief review of the relevant results concerning the CPHD recursion. Denote by $P_{j}^{n}$ the permutation coefficient $n ! /(n-j) !,\langle\cdot, \cdot\rangle$ the inner product defined between two real valued functions $\alpha$ and $\beta$ by $\langle\alpha, \beta\rangle=\int \alpha(x) \beta(x) d x$ (or $\sum_{\ell=0}^{\infty} \alpha(\ell) \beta(\ell)$ when $\alpha$ and $\beta$ are real sequences), and $e_{j}(\cdot)$ the elementary symmetric function of order $j$ defined for a finite set $Z$ of real numbers by $e_{j}(Z)=\sum_{S \subseteq Z,|S|=j} \prod_{\zeta \in S} \zeta$ with $e_{0}(Z)=1$ by convention.

It was established in [15] (Section V-A) that the CPHD recursion for tracking an unknown and time-varying number of targets can be simplified when the number of targets is constant. Let $v_{k \mid k-1}$ and $v_{k}$ be the predicted and posterior intensities respectively at time $k$. If there are no target births nor deaths and $N \in \mathbb{N}$ is the fixed and known number of targets, then the CPHD 
cardinality recursion reduces to $\rho_{k-1}(\cdot)=\rho_{k \mid k-1}(\cdot)=\rho_{k}(\cdot)=$ $\delta_{N}(\cdot)$, and the CPHD intensity recursion reduces to

$$
\begin{aligned}
v_{k \mid k-1}(x)= & \int f_{k \mid k-1}(x \mid \zeta) v_{k-1}(\zeta) d \zeta \\
v_{k}(x)= & \frac{\Upsilon_{k}^{1}\left[v_{k \mid k-1}, Z_{k}\right](N)}{\Upsilon_{k}^{0}\left[v_{k \mid k-1}, Z_{k}\right](N)}\left[1-p_{D, k}(x)\right] v_{k \mid k-1}(x) \\
& +\sum_{z \in Z_{k}} \frac{\Upsilon_{k}^{1}\left[v_{k \mid k-1}, Z_{k} \backslash\{z\}\right](N)}{\Upsilon_{k}^{0}\left[v_{k \mid k-1}, Z_{k}\right](N)} \\
& \times \psi_{k, z}(x) v_{k \mid k-1}(x)
\end{aligned}
$$

where

$$
\begin{aligned}
\Upsilon_{k}^{u}[v, Z](n)= & \sum_{j=0}^{\min (|Z|, n)}(|Z|-j) ! \rho_{K, k}(|Z|-j) P_{j+u}^{n} \\
& \times \frac{\left\langle 1-p_{D, k}, v\right\rangle^{n-(j+u)}}{\langle 1, v\rangle^{n}} e_{j}\left(\Xi_{k}(v, Z)\right) \\
\psi_{k, z}(x)= & \frac{1}{c_{k}(z)} g_{k}(z \mid x) p_{D, k}(x) \\
\Xi_{k}(v, Z)= & \left\{\left\langle v, \psi_{k, z}\right\rangle: z \in Z\right\} .
\end{aligned}
$$

Assuming no extraneous target-generated measurements, the proposed RFS single-target Bayes' recursion reduces to the above mentioned CPHD recursion restricted to a single target. Note that for the purposes of comparison, these particular conditions ensure that both recursions assume the same underlying dynamic and measurement model for the target. The restriction in the CPHD recursion for no target births nor deaths and exactly one target present ensures consistency with the dynamic model in the RFS single-target Bayes' recursion. Indeed, the CPHD recursion for a single target coincides with the multiple hypothesis tracker (MHT) for a single target (see [24]). The restriction in the RFS single-target Bayes' recursion for no extraneous target-generated measurements ensures consistency with the measurement model in the CPHD recursion which accommodates at most one target-generated measurement.

The agreement between these two recursions can be expected for the following reason. Consider the above CPHD recursion and recall its assumption that the target state RFS at any time is an i.i.d. cluster process [14], [15] (see Section II-B for the meaning of an i.i.d. cluster process). Observe that if the cardinality distribution of an i.i.d. cluster process is $\delta_{1}(\cdot)$ (i.e., the value of the process is always a singleton set), then the intensity is the same as the normalized intensity, and hence the intensity is actually the probability density (i.e., the intensity of the RFS is the probability density of the single constituent point of the RFS). If $N=1$ in the above CPHD recursion, (i.e., exactly one target is present at all times), then the CPHD cardinality recursion states that $\rho_{k}(\cdot)=\delta_{1}(\cdot)$ (i.e., the target state RFS is always a singleton), and hence the CPHD intensity recursion for $v_{k}$ actually propagates the probability density $\pi_{k}$ (i.e., the propagated intensity is actually the probability density of the single-target state). Thus, since both the RFS single-target Bayes' recursion and the above CPHD recursion assume the same underlying model, and both recursions propagate the posterior density of the target state, it would be expected that their recursions are consistent. This is indeed true and is stated in the following proposition (See Appendix B for the proof).

Proposition 2: The special case of the proposed RFS singletarget Bayes' recursion (16), (17) with no extraneous target-generated measurements (i.e., (16), (17) with $E_{k}\left(x_{k}\right)=\emptyset$ hence $v_{K, k}(z \mid x)=v_{K, k}(z)=v_{W, k}(z)$ and $c_{k}(z \mid x)=c_{k}(z)=$ $\left.v_{W, k}(z) /\left\langle 1, v_{W, k}\right\rangle\right)$ is identical to the special case of the CPHD recursion (19), (20) with no target births nor deaths and exactly one target present (i.e., (19), (20) with $N=1$ ).

This result establishes that under the common dynamic and measurement model stated above, the proposed derivation of the RFS single-target Bayes' recursion from first principles using point process theory agrees with Mahler's derivation of the CPHD recursion using FISST. This agreement further consolidates the utility and power of FISST.

\section{Sequential Monte Carlo Implementation}

In this section, we describe a generic sequential Monte Carlo (SMC) (see also [19], [25]) implementation of the RFS singletarget Bayes' recursion (16), (17) and demonstrate the proposed filter on a nonlinear tracking example. Note that the proposed SMC implementation inherits the usual convergence properties [26], [27] since the recursion (16), (17) propagates the true posterior density of the target state.

\section{A. Recursion}

Suppose at time $k-1$ that the posterior density $p_{k-1}(\cdot)$ is represented by set of weighted particles $\left\{w_{k-1}^{(i)}, x_{k-1}^{(i)}\right\}_{i=1}^{N}$, i.e.,

$$
p_{k-1}\left(x_{k-1} \mid Z_{1: k-1}\right) \approx \sum_{i=1}^{N} w_{k-1}^{(i)} \delta_{x_{k-1}^{(i)}}\left(x_{k-1}\right) .
$$

Then, for a given proposal density $q_{k}\left(\cdot \mid x_{k-1}^{(i)}, Z_{k}\right)$ satisfying $\operatorname{support}\left(p_{k}\right) \subseteq \operatorname{support}\left(q_{k}\right)$, the particle filter approximates the posterior density $p_{k}(\cdot)$ by a new set of weighted particles $\left\{w_{k}^{(i)}, x_{k}^{(i)}\right\}_{i=1}^{N}$, i.e.

$$
p_{k}\left(x_{k} \mid Z_{1: k}\right) \approx \sum_{i=1}^{N} w_{k}^{(i)} \delta_{x_{k}^{(i)}}\left(x_{k}\right)
$$

where

$$
\begin{aligned}
& x_{k}^{(i)} \sim q_{k}\left(\cdot \mid x_{k-1}^{(i)}, Z_{k}\right) \\
& w_{k}^{(i)}=\tilde{w}_{k}^{(i)} / \sum_{i=1}^{N} \tilde{w}_{k}^{(i)} \\
& \tilde{w}_{k}^{(i)}=w_{k-1}^{(i)} \frac{\eta_{k}\left(Z_{k} \mid x_{k}^{(i)}\right) f_{k \mid k-1}\left(x_{k}^{(i)} \mid x_{k-1}^{(i)}\right)}{q_{k}\left(x_{k}^{(i)} \mid x_{k-1}^{(i)}, Z_{k}\right)} .
\end{aligned}
$$

It can be seen that the proposed algorithm has the same computational complexity as the standard single-target particle filter.

The recursion is initialized by generating a set of weighted particles $\left\{w_{0}^{(i)}, x_{0}^{(i)}\right\}_{i=1}^{N}$ representing $p_{0}$. Equations (26)-(28) then provide a recursion for computing the set of weighted particles representing $p_{k}$ from those representing $p_{k-1}$ when a new measurement arrives. 
Resampling is usually performed after each update to minimize particle degeneracy, and after resampling, an optional Markov chain Monte Carlo (MCMC) step can be used to increase particle diversity (see [19] and [28] for further details).

\section{B. Nonlinear Example}

In this section, a nonlinear scenario is used to demonstrate the performance of the particle implementation of the RFS single-target Bayes' recursion. In particular, a nearly constant turn model with varying turn rate [29] together with bearing and range measurements is considered. The observation region is the half disc of radius $2000 \mathrm{~m}$. The state variable $x_{k}=\left[\tilde{x}_{k}^{T}, \omega_{k}\right]^{T}$ comprises the planar position and velocity $\tilde{x}_{k}^{T}=\left[p_{x, k}, \dot{p}_{x, k}, p_{y, k}, \dot{p}_{y, k}\right]$ as well as the turn rate $\omega_{k}$. The state transition model is

$$
\begin{aligned}
\tilde{x}_{k} & =F\left(\omega_{k-1}\right) \tilde{x}_{k-1}+G w_{k-1} \\
\omega_{k} & =\omega_{k-1}+\Delta u_{k-1}
\end{aligned}
$$

where

$$
F(\omega)=\left[\begin{array}{cccc}
1 & \frac{\sin \omega \Delta}{\omega} & 0 & -\frac{1-\cos \omega \Delta}{\omega} \\
0 & \cos \omega \Delta & 0 & -\sin \omega \Delta \\
0 & \frac{1-\cos \omega \Delta}{\omega} & 1 & \frac{\sin \omega \Delta}{\omega} \\
0 & \sin \omega \Delta & 0 & \cos \omega \Delta
\end{array}\right], G=\left[\begin{array}{cc}
\frac{\Delta^{2}}{2} & 0 \\
\Delta & 0 \\
0 & \frac{\Delta^{2}}{2} \\
0 & \Delta
\end{array}\right]
$$

$w_{k-1} \sim \mathcal{N}\left(\cdot ; 0, \sigma_{w}^{2} I\right)$, and $u_{k-1} \sim \mathcal{N}\left(\cdot ; 0, \sigma_{u}^{2} I\right)$ with $\Delta=1 \mathrm{~s}$, $\sigma_{w}=5 \mathrm{~m} / \mathrm{s}^{2}$, and $\sigma_{u}=\pi / 180 \mathrm{rad} / \mathrm{s}$. The observation region is the half disc $[-\pi / 2, \pi / 2] \mathrm{rad} \times[0,2000] \mathrm{m}$. The primary targetgenerated measurement is a noisy bearing and range vector

$$
z_{k}=\left[\operatorname{atan}\left(p_{x, k} / p_{y, k}\right), \sqrt{p_{x, k}^{2}+p_{y, k}^{2}}\right]^{T}+\varepsilon_{k}
$$

where $\varepsilon_{k} \sim \mathcal{N}\left(\cdot ; 0, R_{k}\right)$, with $R_{k}=\operatorname{diag}\left(\left[\sigma_{\theta}^{2}, \sigma_{r}^{2}\right]^{T}\right), \sigma_{\theta}=$ $2(\pi / 180) \mathrm{rad}$, and $\sigma_{r}=10 \mathrm{~m}$. The sensor field of view is modeled by

$$
p_{D, k}(x)=\frac{\bar{p}_{D, k} \mathcal{N}\left(\left[p_{x, k}, p_{y, k}\right]^{T} ;[250,250]^{T}, 10^{6} I_{2}\right)}{\mathcal{N}\left([0,0]^{T} ;[0,0]^{T}, 10^{6} I_{2}\right)},
$$

where $\bar{p}_{D, k}=0.98$ and $I_{n}$ denotes an $n \times n$ identity matrix. Extraneous measurements are modeled as a Poisson RFS with intensity

$$
\begin{aligned}
v_{E, k}(z \mid x)= & \lambda_{k}^{(1)} \mathcal{N} \\
& \times\left(z ;\left[\operatorname{atan}\left(p_{x, k} / p_{y, k}\right), 2 \sqrt{p_{x, k}^{2}+p_{y, k}^{2}}\right]^{T}, D_{k}\right)
\end{aligned}
$$

where $\lambda_{k}^{(1)}=3, D_{k}=\sigma_{\iota}^{2} I_{2}$ and $\sigma_{\iota}=10 \mathrm{~m}$. Clutter is modeled as a Poisson RFS with intensity

$$
v_{W, k}(z)=\lambda_{k}^{(0)} u(z)
$$

where $u(\cdot)$ is the uniform probability density over the observation region, $\lambda_{k}^{(0)}=\lambda_{c, k} V$ is the expected number of clutter returns with $V=2000 \pi$ (radm) the "volume" of the observation region and $\lambda_{c, k}=3.2 \times 10^{-3}(\mathrm{radm})^{-1}$ (giving an average of $\lambda_{k}^{(0)}=20$ clutter returns per scan).
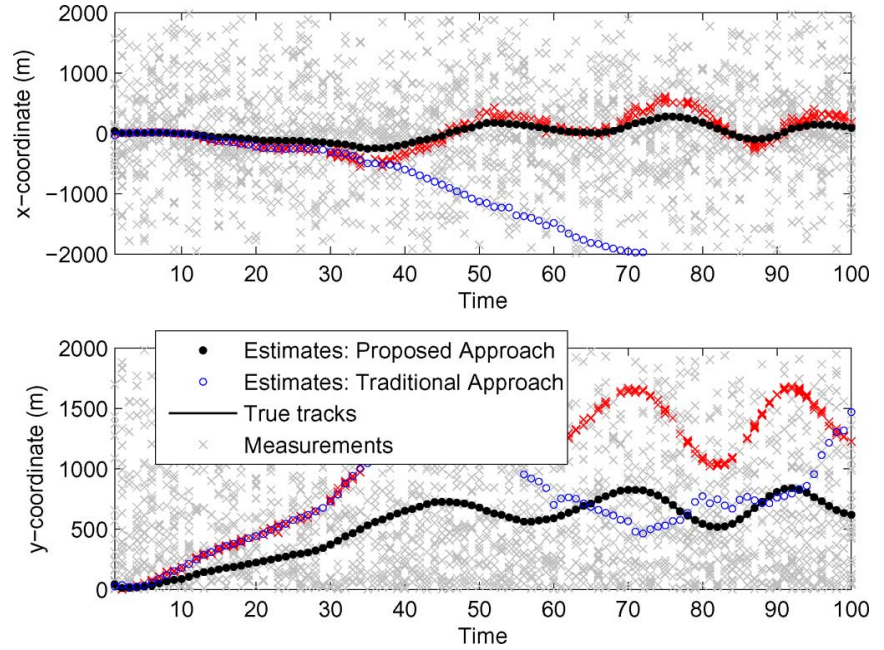

Fig. 1. Estimates from the particle RFS single-target Bayes' filter and traditional approach in $x$ and $y$ coordinates versus time.

At each time step, $N=1000$ particles are used, the transition is used as the proposal, and resampling is performed. The filter is initialized with the following initial prior:

$p_{0}=\mathcal{N}(\cdot ;[0,0.4,0,0.7,0], \operatorname{diag}(2500,25,2500,25,0.0025))$.

Fig. 1 show the tracks, measurements and EAP filter estimates for $x$ and $y$ coordinates versus time on a typical sample run. Note that extraneous target-generated measurements are color coded in red for visual reference. This figure suggests that the proposed filter satisfactorily tracks the target in the presence of multiple measurements generated by the target, clutter and state dependent field of view.

To evaluate the performance of the proposed filter, we compare it with the nonlinear analogue of the Gaussian mixture filter in [4]. Our reason for choosing this filter is that it subsumes many popular traditional techniques for tracking in clutter including the PDA. A typical sample run of this filter on the same set of data is also superimposed on Fig. 1, which suggests that the traditional approach tends to follow the pattern of extraneous target-generated measurements and hence is prone to track loss. This is further reinforced in Fig. 2, which shows the root mean square error (RMSE) and circular position error probability (CPEP) (for a $20 \mathrm{~m}$ error radius) versus clutter rate, for both the proposed filter and the traditional filter. The RMSE and CPEP for each clutter rate is obtained from 1000 Monte Carlo (MC) runs on the same target trajectory but with independently generated measurements for each trial. Fig. 2 suggests that across a wide range of clutter conditions, the proposed RFS single-target Bayes' filter performs better than traditional methods. The former correctly identifies the track, whereas the latter consistently loses track. Moreover, these performance results are consistent with the fact that the nonlinear analogue of the filter in [4] can essentially be considered a special case of the proposed SMC RFS single-target Bayes' recursion in which the observation model assumes $E_{k}\left(x_{k}\right) \equiv \emptyset$ (hence $v_{E, k}(z \mid x)=0$ and $\rho_{E, k}(n \mid x)=\delta_{0}(n)$ ). 

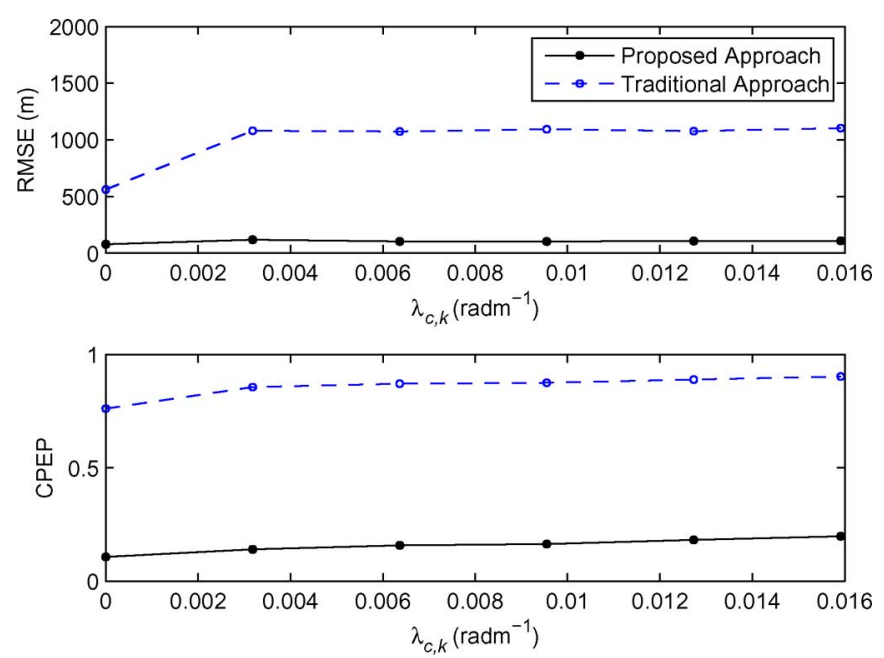

Fig. 2. RMSE and CPEP (20 m error radius) from $1000 \mathrm{MC}$ runs for varying $\lambda_{c, k}$.

\section{ANALYTIC SOLUTION FOR LINEAR GAUSSIAN MODELS}

In this section, we derive a closed-form solution to the RFS single-target Bayes' recursion (16), (17) for the special class of linear Gaussian single-target models. In addition to linear Gaussian transition and likelihood

$$
\begin{aligned}
f_{k \mid k-1}(x \mid \zeta) & =\mathcal{N}\left(x ; F_{k-1} \zeta, Q_{k-1}\right) \\
g_{k}(z \mid x) & =\mathcal{N}\left(z ; H_{k} x, R_{k}\right)
\end{aligned}
$$

the linear Gaussian single-target model assumes a constant sensor field of view, i.e., $p_{D, k}(x)=p_{D, k}$ and linear Gaussian intensity of extraneous target-generated measurements, i.e.,

$$
\begin{aligned}
v_{E, k}(z \mid x) & =\lambda_{k}^{(1)} c_{k}^{(1)}(z \mid x) \\
c_{k}^{(1)}(z \mid x) & =\mathcal{N}\left(z ; B_{k} x+b_{k}, D_{k}\right)
\end{aligned}
$$

where $\lambda_{k}^{(1)}$ is the expected number of extraneous target-generated observations and $c_{k}^{(1)}(\cdot \mid \cdot)$ is the likelihood of individual extraneous observations at time $k, B_{k}$ is the extraneous observation matrix, $b_{k}$ is a constant vector, and $D_{k}$ is the extraneous observation covariance at time $k$.

Observe that if clutter has intensity

$$
v_{W, k}(z)=\lambda_{k}^{(0)} c_{k}^{(0)}(z)
$$

where $\lambda_{k}^{(0)}$ is the mean clutter rate and $c_{k}^{(0)}(\cdot)$ is the density of clutter at time $k$, then, $K_{k}\left(x_{k}\right)$ in (9) is a Poisson RFS with intensity $v_{K, k}(z \mid x)=v_{E, k}(z \mid x)+v_{W, k}(z)$. Hence, the cardinality distribution of $K_{k}\left(x_{k}\right)$ is Poisson with rate $\lambda_{k}^{(0)}+\lambda_{k}^{(1)}$ and individual elements of $K_{k}\left(x_{k}\right)$ are i.i.d. according to the probability density

$$
c_{k}(z \mid x)=w_{c, k}^{(0)} c_{k}^{(0)}(z)+w_{c, k}^{(1)} \mathcal{N}\left(z ; B_{k} x+b_{k}, D_{k}\right)
$$

where $w_{c, k}^{(i)}=\lambda_{k}^{(i)} /\left(\lambda_{k}^{(0)}+\lambda_{k}^{(1)}\right)$ for $i=0,1$. Note that results of this section can be easily extended to the case where $v_{E, k}$ is a Gaussian mixture as outlined in Section V-C.

\section{A. Closed-Form Recursion}

The following propositions establish an exact closed-form solution to the recursion (16), (17) for the linear Gaussian singletarget model.

Proposition 3: If at time $k-1$ the posterior density $p_{k-1}(\cdot)$ is a Gaussian mixture of the form

$$
p_{k-1}(x)=\sum_{j=1}^{J_{k-1}} w_{k-1}^{(j)} \mathcal{N}\left(x ; m_{k-1}^{(j)}, P_{k-1}^{(j)}\right)
$$

then the predicted density $p_{k \mid k-1}(\cdot)$ is also a Gaussian mixture and is given by

$$
p_{k \mid k-1}(x)=\sum_{i=1}^{J_{k-1}} w_{k-1}^{(i)} \mathcal{N}\left(x ; m_{k \mid k-1}^{(i)}, P_{k \mid k-1}^{(i)}\right)
$$

where

$$
\begin{aligned}
m_{k \mid k-1}^{(i)} & =F_{k-1} m_{k-1}^{(i)} \\
P_{k \mid k-1}^{(i)} & =Q_{k-1}+F_{k-1} P_{k-1}^{(i)} F_{k-1}^{T} .
\end{aligned}
$$

For the closed-form update equation, it is convenient to define two intermediate operators $G_{k, z}$ and $C_{k, z}$ on the state space $\mathcal{X}$ by

$$
\begin{aligned}
\left(G_{k, z} \phi\right)(x) & =\Xi_{z}\left[g_{k}, \phi\right](x) \\
\left(C_{k, z} \phi\right)(x) & =\Xi_{z}\left[c_{k}, \phi\right](x)
\end{aligned}
$$

where $\left(\Xi_{z}[s, \phi]\right)(x)=s(z \mid x) \phi(x)$. Note that if

$$
\begin{gathered}
s(z \mid x)=\bar{s}(z)+w_{s} \mathcal{N}\left(z ; H_{s} x+b_{s}, P_{s}\right) \\
\phi(x)=\sum_{u=1}^{U} w_{\phi}^{(u)} \mathcal{N}\left(x ; m_{\phi}^{(u)}, P_{\phi}^{(u)}\right)
\end{gathered}
$$

then $\left(\Xi_{z}[s, \phi]\right)(\cdot)$ is a Gaussian mixture and is given by

$$
\left(\Xi_{z}[s, \phi]\right)(x)=\bar{s}(z) \phi(x)+\sum_{u=1}^{U} w_{\Xi}^{(u)}(z) \mathcal{N}\left(x ; m_{\Xi}^{(u)}(z), P_{\Xi}^{(u)}\right)
$$

where

$$
\begin{aligned}
w_{\Xi}^{(u)}(z) & =w_{s} w_{\phi}^{(u)} q_{\Xi}^{(u)}(z) \\
q_{\Xi}^{(u)}(z) & =\mathcal{N}\left(z ; H_{s} m_{\phi}^{(u)}+b_{s}, P_{s}+H_{s} P_{\phi}^{(u)} H_{s}^{T}\right) \\
m_{\Xi}^{(u)}(z) & =m_{\phi}^{(u)}+K_{\Xi}^{(u)}\left(z-H_{s} m_{\phi}^{(u)}-b_{s}\right) \\
P_{\Xi}^{(u)} & =\left(I-K_{\Xi}^{(u)} H_{s}\right) P_{\phi}^{(u)} \\
K_{\Xi}^{(u)} & =P_{\phi}^{(u)} H_{s}^{T}\left(P_{s}+H_{s} P_{\phi}^{(u)} H_{s}^{T}\right)^{-1} .
\end{aligned}
$$


Proposition 4: If at time $k$ the predicted density $p_{k \mid k-1}(\cdot)$ is a Gaussian mixture of the form

$$
p_{k \mid k-1}(x)=\sum_{j=1}^{J_{k \mid k-1}} w_{k \mid k-1}^{(j)} \mathcal{N}\left(x ; m_{k \mid k-1}^{(j)}, P_{k \mid k-1}^{(j)}\right),
$$

then, $\eta_{k}\left(Z_{k} \mid x\right) p_{k \mid k-1}(x)$ is also a Gaussian mixture and is given by

$$
\eta_{k}\left(Z_{k} \mid x\right) p_{k \mid k-1}(x)=\bar{d}_{k}(x)+\sum_{z^{*} \in Z_{k}} d_{k}\left(x ; z^{*}\right)
$$

where

$$
\begin{aligned}
\bar{d}_{k}(x)= & \rho_{K, k}\left(\left|Z_{k}\right|\right) \cdot\left|Z_{k}\right| ! \cdot\left(1-p_{D, k}\right) \\
& \times\left(\left[\prod_{z \in Z_{k}} C_{k, z}\right] p_{k \mid k-1}\right)(x) \\
d_{k}\left(x ; z^{*}\right)= & \rho_{K, k}\left(\left|Z_{k}\right|-1\right) \cdot\left(\left|Z_{k}\right|-1\right) ! \cdot p_{D, k} \\
& \times\left(\left[\prod_{z \neq z^{*}} C_{k, z}\right]\left[G_{k, z^{*}} p_{k \mid k-1}\right]\right)(x)
\end{aligned}
$$

and by convention, a product of operators denotes a composition, i.e., $\prod_{i=1}^{N(k)} C_{k, z_{k, i}}=C_{k, z_{k, 1}} \circ C_{k, z_{k, 2}} \circ \cdots \circ C_{k, z_{k, N(k)}}$.

Remark: The mixture (50) can also be written as

$$
\eta_{k}\left(Z_{k} \mid x\right) p_{k \mid k-1}(x)=\sum_{j=1}^{J_{k}} w_{k}^{(j)} \mathcal{N}\left(x ; m_{k}^{(j)}, P_{k}^{(j)}\right) .
$$

Consequently, the posterior density is given by

$$
p_{k}(x)=\sum_{j=1}^{J_{k}} \tilde{w}_{k}^{(j)} \mathcal{N}\left(x ; m_{k}^{(j)}, P_{k}^{(j)}\right)
$$

where $\tilde{w}_{k}^{(j)}=w_{k}^{(j)} / \sum_{j=1}^{J_{k}} w_{k}^{(j)}$ and $\sum_{j=1}^{J_{k}} w_{k}^{(j)}$ is the normalizing constant in the RFS single-target Bayes' recursion.

Remark: Proposition 4 can be interpreted as follows: given that the predicted density $p_{k \mid k-1}$ is a Gaussian mixture, then the posterior density $p_{k}$ is also a Gaussian mixture comprised of the mixtures $\bar{d}_{k}(\cdot)$ and $d_{k}\left(\cdot ; z^{*}\right)$ for each $z^{*} \in Z_{k}$. The mixture $\bar{d}_{k}(\cdot)$ is obtained by recursively applying $C_{k, z}$ starting with $p_{k \mid k-1}$ for each $z \in Z_{k}$. The mixture $d_{k}\left(\cdot ; z^{*}\right)$ is obtained by applying $G_{k, z^{*}}$ once to $p_{k \mid k-1}$ and then recursively applying $C_{k, z}$ for each $z \in Z_{k} \backslash\left\{z^{*}\right\}$.

Remark: The above closed-form solution can be extended to accommodate a state dependent sensor field of view. For an exponential mixture form of $p_{D, k}(\cdot)$, a closed-form update can easily be obtained following the approach in [13], though this extension will not be shown here due to space constraints.

It follows by induction from Propositions 3 and 4 that if the initial density $p_{0}$ is a Gaussian mixture, then all subsequent predicted $p_{k \mid k-1}$ and posterior densities $p_{k}$ are also Gaussian mixtures. Proposition 3 provides closed-form expressions for computing the weights, means and covariances of $p_{k \mid k-1}$, whilst Proposition 4 provides closed-form expressions for computing the weights, means and covariances of $p_{k}$ when a new set of measurements arrives. Note that Proposition 3 is the prediction step of the Gaussian sum filter [30], whilst the proof for Proposition 4 is nontrivial; see Appendix $\mathrm{C}$ for further details.

\section{B. Implementation Issues}

In the proposed closed-form recursion, if the posterior at time $k-1$ has $J_{k-1}$ mixture components, then the posterior at time $k$ has

$$
J_{k-1}\left(2^{\left|Z_{k}\right|}+\left|Z_{k}\right| 2^{\left|Z_{k}\right|-1}\right)=\mathcal{O}\left(J_{k-1} \cdot 2^{\left|Z_{k}\right|}\right)
$$

mixture components. Our closed-form solution has the same complexity as the traditional Gaussian mixture approach in [4]. Note that the exponential growth of mixture components with the number of received measurements results in an exponential growth of mixture components with time. It can be seen that the number of mixture components required to represent the posterior exactly increases without bound. Thus, the closed-form solution does not necessarily guarantee computational tractability, and additional approximations to reduce the number of Gaussian components are needed. A Rao-Blackwellized particle filter [31] (that exploits the closed-form solution) can be employed as a random strategy for reducing the number of Gaussian components. In this paper, we consider simpler strategies for mitigating this problem.

1) Gating: To reduce the number of measurements that the filter has to process, a standard measurement validation technique [3] can be used before performing the update at each time step. The idea is to only retain measurements that are 'close' to the predicted measurement. Gating, however, also reduces the ability of the filter to detect targets.

Along the lines of [3], if at time $k$ the predicted density $p_{k \mid k-1}$ is given and is of the form (49), define the validation region (or prediction gate) of the $i$ th predicted mixture component for a threshold value $\delta$ by

$$
V_{k}^{(i)}(\delta)=\left\{z:\left(z-\eta_{k \mid k-1}^{(i)}\right)^{T}\left[S_{k}^{(i)}\right]^{-1}\left(z-\eta_{k \mid k-1}^{(i)}\right)<\delta\right\}
$$

where $\eta_{k \mid k-1}^{(i)}=H_{k} x_{k \mid k-1}^{(i)}$ and $S_{k}^{(i)}=R_{k}+H_{k} P_{k \mid k-1}^{(i)} H_{k}^{T}$ are the predicted measurement and innovation covariance for the $i$ th component respectively, and $\delta \sim \chi_{n_{z}}^{2}$ is chosen a threshold parameter (note $\sqrt{\delta}$ is interpreted as the number of sigmas or standard deviations of the gate). Then, the measurement set given to the filter $Z_{k}^{\prime}$ is comprised of those measurements falling within the combined validation region, i.e.,

$$
Z_{k}^{\prime}=Z_{k} \cap\left[\bigcup_{i=1}^{J_{k \mid k-1}} V_{k}^{(i)}(\delta)\right] .
$$

2) Managing Mixture Components: First, to limit the growth of the number of mixture components with the number of received measurements, the following approach can be used. Since this growth is caused by recursive application of the operators $G_{k, z}$ and/or $C_{k, z}$ (in the calculation of the mixtures $d_{k}(\cdot)$ and $\left.\bar{d}_{k}(\cdot)\right)$, a simple way to limit this growth is to truncate each intermediate result during the update at each time $k$. In other words, having applied $G_{k, z}$ or $C_{k, z}$ to the mixture $\phi(\cdot)$, the resultant mixture $\left(G_{k, z} \phi\right)(x)=\Xi_{z}\left[g_{k}, \phi\right](x)$ 
or $\left(C_{k, z} \phi\right)(x)=\Xi_{z}\left[c_{k}, \phi\right](x)$ is approximated by a truncated version, i.e., each resultant mixture (58) is approximated by

$$
\left(\Xi_{z}[s, \phi]\right)(x) \approx \sum_{u \in \hat{I}_{k}} \hat{w}_{s}^{(u)}(z) \mathcal{N}\left(x ; m_{s}^{(u)}(z), P_{s}^{(u)}\right)
$$

where $\hat{I}_{k} \subseteq\{1, \ldots, U\}$ is the set of indexes of components that are retained in the approximation (e.g., $I_{k}$ contains the indexes of the $\hat{J}_{\max }$ components or of the top $10 \%$ of components with the highest weights), and $\hat{w}_{s}^{(u)}(z)=w_{s}^{(u)}(z) \sum_{u=1}^{U} w_{s}^{(u)} / \sum_{u \in \hat{I}_{k}} \hat{w}_{s}^{(u)}(z)$ ensures that the sum of the weights before and after truncation are the same.

Second, to limit the growth of the number of components with time, a standard pruning and merging procedure given in [13] can be used, which is summarized as follows. If at time $k$ the posterior density $p_{k}$ is given and is of the form (54), it is approximated by a pruned and merged version

$$
\hat{p}_{k}(x) \approx \sum_{j=1}^{J_{\max }} \hat{w}_{k}^{(j)} \mathcal{N}\left(x ; \hat{m}_{k}^{(j)}, \hat{P}_{k}^{(j)}\right)
$$

in which components with weights below a threshold $T$ are discarded, components with peaks within a distance $U$ of each other are merged, and only the $J_{\max }$ components with the highest weights are retained. See [13] for the exact meaning of these parameters and full details on implementation.

\section{Extension to Gaussian Mixture Form $v_{E, k}$}

The closed-form solution given by Propositions 3 and 4 can be easily extended to accommodate the case where $v_{E, k}(\cdot \cdot \cdot)$ is a Gaussian mixture of the form

$$
v_{E, k}(z \mid x)=\sum_{i=1}^{J_{E, k}} \lambda_{k}^{(i)} \mathcal{N}\left(z ; B_{k}^{(i)} x+b_{k}^{(i)}, D_{k}^{(i)}\right)
$$

as follows. First, note that $v_{K, k}(z \mid x)=v_{W, k}(z)+v_{E, k}(z \mid x)$ and hence

$$
c_{k}(z \mid x)=w_{c, k}^{(0)} c_{k}^{(0)}(z)+\sum_{i=1}^{J_{E, k}} w_{c, k}^{(i)} \mathcal{N}\left(z ; B_{k}^{(i)} x+b_{k}^{(i)}, D_{k}^{(i)}\right)
$$

where $w_{c, k}^{(i)}=\lambda_{k}^{(i)} / \sum_{j=1}^{J_{E, k}} \lambda_{k}^{(j)}$ for $i=0,1, \ldots, J_{E, k}$. Observe then that the result of Proposition 4 can be extended by providing a closed-form expression for $\left(C_{k, z} \phi\right)(x)=\Xi_{z}\left[c_{k}, \phi\right](x)$ where $\left(\Xi_{z}[s, \phi]\right)(x)=s(z \mid x) \phi(x)$ as follows. Note that if

$$
s(z \mid x)=\bar{s}(z)+\sum_{i=1}^{I} w_{s}^{(i)} \mathcal{N}\left(z ; H_{s}^{(i)} x+b_{s}^{(i)}, P_{s}^{(i)}\right)
$$

and $\phi(x)$ is of the form (42), then the expression for $\Xi[s, \phi](\cdot)$ is still a Gaussian mixture and becomes

$$
\begin{aligned}
\left(\Xi_{z}[s, \phi]\right) & (x)=\bar{s}(z) \phi(x) \\
& +\sum_{i=1}^{I} \sum_{u=1}^{U} w_{\Xi}^{(i, u)}(z) \mathcal{N}\left(x ; m_{\Xi}^{(i, u)}(z), P_{\Xi}^{(i, u)}\right)
\end{aligned}
$$

where

$$
\begin{aligned}
w_{\Xi}^{(i, u)}(z) & =w_{s}^{(i)} w_{\phi}^{(u)} q_{\Xi}^{(u)}(z) \\
q_{\Xi}^{(i, u)}(z) & =\mathcal{N}\left(z ; H_{s}^{(i)} m_{\phi}^{(u)}+b_{s}^{(i)}, P_{s}^{(i)}+H_{s}^{(i)} P_{\phi}^{(u)}\left[H_{s}^{(i)}\right]^{T}\right) \\
m_{\Xi}^{(i, u)}(z) & =m_{\phi}^{(u)}+K_{\Xi}^{(i, u)}\left(z-H_{s}^{(i)} m_{\phi}^{(u)}-b_{s}^{(i)}\right) \\
P_{\Xi}^{(i, u)} & =\left(I-K_{\Xi}^{(i, u)} H_{s}^{(i)}\right) P_{\phi}^{(u)} \\
K_{\Xi}^{(i, u)} & =P_{\phi}^{(u)}\left[H_{s}^{(i)}\right]^{T}\left(P_{s}^{(i)}+H_{s}^{(i)} P_{\phi}^{(u)}\left[H_{s}^{(i)}\right]^{T}\right)^{-1} .
\end{aligned}
$$

\section{Linear Gaussian Example}

In this section, a linear Gaussian scenario is used to demonstrate the performance of the closed-form implementation of the RFS single-target Bayes' recursion. The following linear Gaussian single-target model is used. The target state is a vector of position and velocity $x_{k}=\left[p_{x, k}, p_{y, k}, \dot{p}_{x, k}, \dot{p}_{y, k}\right]^{T}$ that follows the linear Gaussian transition model (29) with

$$
F_{k}=\left[\begin{array}{cc}
I_{2} & \Delta I_{2} \\
0_{2} & I_{2}
\end{array}\right], \quad Q_{k}=\sigma_{\nu}^{2}\left[\begin{array}{cc}
\frac{\Delta^{4}}{4} I_{2} & \frac{\Delta^{3}}{2} I_{2} \\
\frac{\Delta^{3}}{2} I_{2} & \Delta^{2} I_{2}
\end{array}\right]
$$

where $I_{n}$ and $0_{n}$ denote the $n \times n$ identity and zero matrices respectively, $\Delta=1 \mathrm{~s}$ is the sampling period, and $\sigma_{\nu}=5\left(\mathrm{~m} / \mathrm{s}^{2}\right)$ is the standard deviation of the process noise. The primary targetgenerated measurement likelihood is linear Gaussian (30) with

$$
H_{k}=\left[\begin{array}{ll}
I_{2} & 0_{2}
\end{array}\right], \quad R_{k}=\sigma_{\varepsilon}^{2} I_{2}
$$

where $\sigma_{\varepsilon}=10 \mathrm{~m}$ is the standard deviation of the measurement noise. The observation region is the square $\mathcal{Z}=[-1000,1000] \times[-1000,1000]$ (units are in meters). The corresponding probability of detection is fixed at $p_{D, k}=0.98$. Extraneous target-generated measurements are modeled as a Poisson RFS with linear Gaussian intensity (31)

$$
v_{E, k}(z \mid x)=\lambda_{k}^{(1)} \mathcal{N}\left(z ; B_{k} x, D_{k}\right)
$$

where $\lambda_{k}^{(1)}=3, B_{k}=\left[2 I_{2} 0_{2}\right], D_{k}=\sigma_{\iota}^{2} I_{2}$ and $\sigma_{\iota}=10 \mathrm{~m}$. Clutter is modeled as a Poisson RFS with intensity

$$
v_{W, k}(z)=\lambda_{k}^{(0)} u(z)
$$

where $u(\cdot)$ is the uniform probability density over $\mathcal{Z}, \lambda_{k}^{(0)}=$ $\lambda_{c, k} V, \lambda_{c, k}=1.25 \times 10^{-5} \mathrm{~m}^{-2}$ is the average clutter intensity and $V=4 \times 10^{6} \mathrm{~m}^{2}$ is the "volume" of $\mathcal{Z}$ (giving an average of $\lambda_{k}^{(0)}=50$ clutter returns per scan).

The gating and pruning/merging procedures described in Section V-B are used. Gating is performed at each time step using a 99\% validation gate. ${ }^{3}$ Pruning and merging is performed at each time step using a weight threshold of $T=10^{-5}$, a merging threshold of $U=4 \mathrm{~m}$, a maximum of $\hat{J}_{\max }=100$

\footnotetext{
${ }^{3}$ The region centered on the predicted measurement with a 0.99 probability of containing a primary target generated measurement.
} 

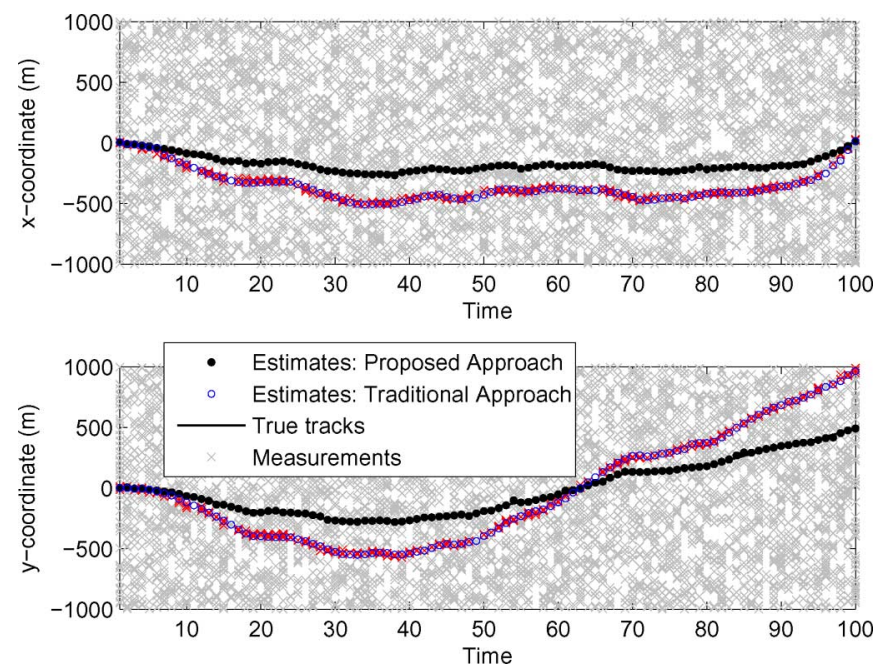

Fig. 3. Estimates from the linear Gaussian RFS single-target Bayes' filter and traditional approach in $x$ and $y$ coordinates versus time.

intermediate components and a maximum of $J_{\max }=100$ overall components.

In this scenario, the target follows a curved path with varying velocity. The filter is initialized with

$$
p_{0}=\mathcal{N}\left(\cdot ;[0,0.4,0,0.7]^{T}, \operatorname{diag}\left(10^{4}, 10^{2}, 10^{4}, 10^{2}\right)\right) .
$$

Fig. 3 illustrates a typical sample run showing the tracks, measurements, $x$ and $y$ coordinates of the maximum a posteriori (MAP) filter estimates versus time. Note that extraneous targetgenerated measurements are color coded in red for visual reference. Note also that the solid line of the true track is difficult to see from this figure as the estimates of the proposed approach are right on top of the true track. This figure suggests that our proposed filter correctly identifies the track and does not suffer from any track loss in the presence of multiple measurements generated by the target and clutter.

Similar to the nonlinear example, for performance comparison purposes, we compare with the Gaussian mixture filter in [4]. Again, our reason for choosing this filter is that it subsumes many popular traditional techniques for tracking in clutter including the PDA. Fig. 3 has superimposed a typical sample run for the same data. It can be seen that the traditional approach tends to lose the track and erroneously follow the pattern of the extraneous target-generated measurements. This observation is supported by the results of $1000 \mathrm{MC}$ runs performed on the same target trajectory but with independently generated measurements for each trial. In Fig. 4, the MC average RMSE and CPEP (for 20-m error radius) are shown versus clutter rate for our proposed filter and for the filter in [4], which suggests that the proposed RFS single-target Bayes' filter performs better than traditional methods. The former correctly identifies the true tracks, whereas the latter consistently loses the true track. Again, these performance results are consistent with the fact that the filter in [4] can essentially be considered a special case of the proposed closed-form linear Gaussian RFS single-target Bayes' recursion in which the observation model assumes $E_{k}\left(x_{k}\right) \equiv \emptyset$ (hence $v_{E, k}(z \mid x)=0$ and $\rho_{E, k}(n \mid x)=\delta_{0}(n)$ ).
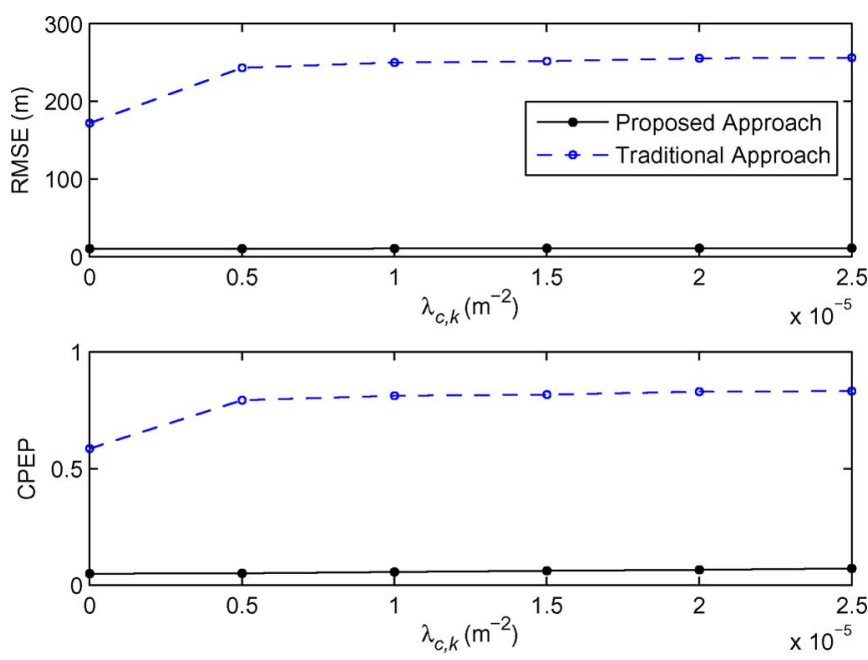

Fig. 4. RMSE and CPEP (20-m error radius) values from $1000 \mathrm{MC}$ runs for varying $\lambda_{c, k}$.

\section{EXTENSION TO NONLINEAR GAUSSIAN MODELS}

In this section, we outline two extensions of the closed-form Gaussian mixture implementation of Section V to accommodate mild nonlinearities using linearization and unscented transforms. Here, the form of the dynamical and measurement models given by the transition density $f_{k \mid k-1}(\cdot \mid \cdot)$ and the likelihood $g_{k}(\cdot \mid \cdot)$ are relaxed to nonlinear Gaussian models

$$
\begin{aligned}
x_{k} & =\varphi_{k}\left(x_{k-1}, \nu_{k-1}\right) \\
z_{k}^{*} & =h_{k}\left(x_{k}, \varepsilon_{k}\right)
\end{aligned}
$$

where $\varphi_{k}$ and $h_{k}$ are the nonlinear state and measurement functions respectively, and $\nu_{k-1}$ and $\varepsilon_{k}$ are independent zero-mean Gaussian noise processes with covariance matrices $Q_{k-1}$ and $R_{k}$ respectively. Additionally, the form of the extraneous target measurements model given by the likelihood of extraneous target measurements $c_{k}^{(1)}(\cdot \mid \cdot)$ is relaxed to a nonlinear function in the state and noise variable

$$
z_{k}=i_{k}\left(x_{k}, \iota_{k}\right)
$$

where $i_{k}$ is the nonlinear extraneous target measurement function and $\iota_{k}$ is an independent zero-mean Gaussian noise process with covariance matrix $D_{k}$.

Analogous to the extended Kalman filter (EKF) [32], [33], the nonlinear prediction and update can be approximated by linearizing $\varphi_{k}, h_{k}, i_{k}$.

Analogous to the unscented Kalman filter (UKF) [34], a nonlinear approximation to the prediction and update can be obtained using the unscented transform (UT). The strategy here is to use the UT to propagate the first and second moments of each mixture component of $p_{k-1}$ and $p_{k \mid k-1}$ through the nonlinear transformations $\varphi_{k}, h_{k}, i_{k}$.

\section{A. Nonlinear Gaussian Example}

In this section, a nonlinear example is used to demonstrate the performance of the EK and UK approximations to the closedform implementation of the RFS single-target Bayes' recursion. The same motion and measurement model as in Section IV-B is used, except that the probability of detection is fixed at $p_{D, k}=$ 

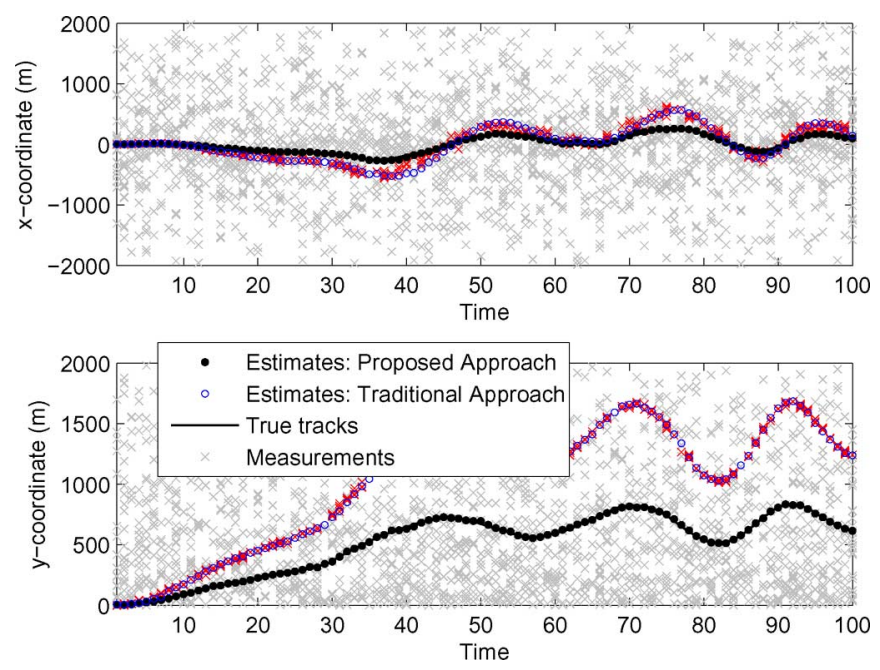

Fig. 5. Estimates from the EK RFS single-target Bayes' filter and traditional approach in $x$ and $y$ coordinates versus time.
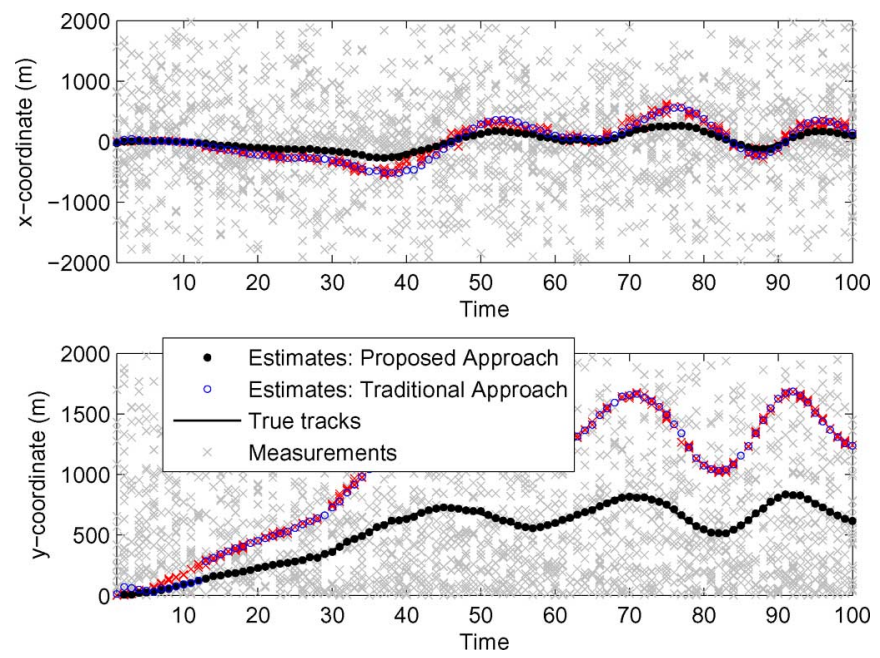

Fig. 6. Estimates from the UK RFS single-target Bayes' filter and traditional approach in $x$ and $y$ coordinates versus time.

0.98. The gating and pruning/merging procedure of Section V-B is used with the same parameters as given in the linear Gaussian demonstrations of Section V-D. The following initial prior is used

$p_{0}=\mathcal{N}(\cdot ;[0,0.4,0,0.7,0], \operatorname{diag}(2500,25,2500,25,0.0025))$.

Figs. 5 and 6 illustrate a typical sample run of the EK and UK approximations, respectively, showing the tracks, measurements, and filter estimates for $x$ and $y$ coordinates versus time. Note that extraneous target-generated measurements are color coded in red for visual reference. These figures show that the proposed EK and UK approximations exhibit similar performance, and are able to satisfactorily accommodate the nonlinear motion and measurement models. Similar to the linear Gaussian example, we also compare with the EK and UK versions of the Gaussian mixture filter in [4]. A typical sample run for these filters is superimposed on Figs. 5 and 6, respectively. Similar to previous experiments, these results further suggest that our proposed approach performs better than traditional methods.

\section{CONCLUSION}

This paper has presented a novel and mathematically rigorous Bayes' recursion that formally accommodates multiple measurements generated by the target, state dependent sensor field of view, and clutter. It was shown that the special case of the proposed recursion with no extraneous target-generated measurements is indeed Mahler's CPHD recursion restricted to the single-target case. A particle implementation has been given, and a closed-form solution has been derived for linear Gaussian models with constant sensor field of view though extensions to exponential mixture sensor field of view are easily derived. The closed-formed solution has also been extended to nonlinear Gaussian models via linearizations and unscented transformations. The complexity of the particle implementation is the same as that of the standard particle filter. The closed-form solution does not necessarily guarantee computational tractability and additional approximations are needed for implementation, analogous to the case of the Gaussian sum filter. Simulations have suggested that the proposed approach outperforms traditional techniques in terms of track loss and localization error.

In light of the proposed Bayesian RFS formulation, all information about the target is encapsulated by the posterior density in a mathematically consistent manner. Hence, it is now possible to study how clutter and detection uncertainty affect tracking performance in the context of Shannon information. From an applications point of view, our formulation is directly applicable to tracking with multiple sensors. Moreover, the provisions for nonconstant sensor field of view, multiple measurements generated by the target, and clutter means that our approach can be adapted for distributed fusion and tracking in sensor networks.

\section{APPENDIX A}

This section derives the likelihood (12) corresponding to the RFS measurement (8) using standard measure theoretic probability. The main steps of the derivation are summarized first. To begin, the probability distribution of $Z_{k}$ conditioned on $x_{k}$ is derived from first principles. Then, the probability density of $Z_{k}$ conditioned on $x_{k}$ is derived by taking the Radon-Nikodým derivative of the corresponding probability distribution with respect to an appropriate reference measure. This probability density is indeed the expression we seek, i.e., the probability density of $Z_{k}$ given $x_{k}$ is the likelihood of the measurement set $Z_{k}$ for a given state $x_{k}$.

Recall that for any Borel subset $\mathcal{S} \subseteq F(Z)$, the probability distribution of $Z_{k}$ given $x_{k}$ is

$$
P_{k}\left(\mathcal{S} \mid x_{k}\right) \equiv \operatorname{Pr}\left(Z_{k} \in \mathcal{S} \mid x_{k}\right) .
$$

Decomposing $\mathcal{S}$ into $\mathcal{S}=\uplus_{r=0}^{\infty} \mathcal{S}_{r}$ where $\mathcal{S}_{r}$ denotes the subset of $\mathcal{S}$ with exactly $r$ elements, the law of total probability gives

$$
P_{k}\left(\mathcal{S} \mid x_{k}\right)=\sum_{r=0}^{\infty} P_{k}\left(\mathcal{S}_{r} \mid x_{k}\right)
$$


Expressions for $P_{k}\left(\mathcal{S}_{r} \mid x_{k}\right)=\operatorname{Pr}\left(Z_{k} \in \mathcal{S}_{r} \mid x_{k}\right)$ are derived as follows. Notice that the observation set can be written explicitly as $\left\{z_{k, 1}, \ldots, z_{k, r}\right\}$. For each time $k$, define the events

$$
\epsilon_{k, r}^{(i)}=\left\{\begin{array}{cl}
\left\{\left|Z_{k}\right|=r, Z_{k}=K_{k}\left(x_{k}\right)\right\} & i=0 \\
\left\{\left|Z_{k}\right|=r, \Theta_{k}\left(x_{k}\right)=\left\{z_{k, i}\right\},\right. & \\
\left.K_{k}\left(x_{k}\right)=Z_{k} \backslash\left\{z_{k, i}\right\}\right\} & i=1, \ldots, r .
\end{array}\right.
$$

Since the events $\epsilon_{k, r}^{(i)}$ for $i=0, \ldots, r$ form a partitioning of the event $Z_{k} \in \mathcal{S}_{r}$, using the law of total probability $P_{k}\left(\mathcal{S}_{r} \mid x_{k}\right)$ can be written as

$$
P_{k}\left(\mathcal{S}_{r} \mid x_{k}\right)=\sum_{i=0}^{r} P\left(\epsilon_{k, r}^{(i)}\right) P_{k}\left(\mathcal{S}_{r} \mid \epsilon_{k, r}^{(i)}, x_{k}\right) .
$$

From the definition in (A3), the probabilities $P\left(\epsilon_{k, r}^{(i)}\right)$ can be evaluated as shown. For $i=0$, if $Z_{k}=K_{k}\left(x_{k}\right)$, then there must be a missed primary measurement detection and $\left|K_{k}\left(x_{k}\right)\right|=r$; thus $P\left(\epsilon_{k, r}^{(0)}\right)$ is given by the probability of a missed primary measurement detection, the cardinality distribution of $K_{k}\left(x_{k}\right)$ evaluated at $r$, and a factorial term to account for the $r$ ! possible permutations of the measurement set, resulting in (A5). For $i \neq 0$, if $\Theta_{k}\left(x_{k}\right)=\left\{z_{k, i}\right\}$, then there is a primary measurement detection and $\left|K_{k}\left(x_{k}\right)\right|=r-1$; thus $P\left(\epsilon_{k, r}^{(i)}\right)$ is given by the probability of a primary measurement detection, the cardinality distribution of $K_{k}\left(x_{k}\right)$ evaluated at $r-1$, and a factorial term to account for the $(r-1)$ ! possible permutations of the measurement set, resulting in (A6). That is

$$
\begin{aligned}
& P\left(\epsilon_{k, r}^{(0)}\right)=\left[1-p_{D, k}\left(x_{k}\right)\right] \rho_{K, k}\left(r \mid x_{k}\right)(r !) \\
& P\left(\epsilon_{k, r}^{(i)}\right)=p_{D, k}\left(x_{k}\right) \rho_{K, k}\left(r-1 \mid x_{k}\right)(r-1) !
\end{aligned}
$$

where $p_{D, k}(\cdot)$ is the probability of detection and $\rho_{K, k}(\cdot \mid \cdot)$ is the cardinality distribution of the RFS $K_{k}\left(x_{k}\right)$.

Also, the probability distributions $P_{k}\left(\mathcal{S}_{r} \mid \epsilon_{k, r}^{(i)}, x_{k}\right)=$ $\operatorname{Pr}\left(Z_{k} \in \mathcal{S}_{r} \mid \epsilon_{k, r}^{(i)}, x_{k}\right)$ can be evaluated as shown. Recall by assumption that the RFS $\Theta_{k}\left(x_{k}\right)$ is a binary RFS, the RFS $K_{k}\left(x_{k}\right)$ is a Poisson RFS, and that these RFSs are independent. Also recall that a measurement from $\Theta_{k}\left(x_{k}\right)$ follows the likelihood $g_{k}\left(\cdot \mid x_{k}\right)$ and that measurements from $K_{k}\left(x_{k}\right)$ are i.i.d. according to $c_{k}\left(\cdot \mid x_{k}\right)$. For $i=0$, given that all $r$ measurements are from $K_{k}\left(x_{k}\right), P_{k}\left(\mathcal{S}_{r} \mid \epsilon_{k, r}^{(0)}, x_{k}\right)$ is obtained by appropriately integrating over $\mathcal{S}_{r}$ the density $\prod_{k=1}^{r} c_{k}\left(z_{k, j} \mid x_{k}\right)$ (the product of the densities of each of the points), resulting in (A7). For $i \neq 0$, given that the $i$ th measurement is from $\Theta_{k}\left(x_{k}\right)$ and independent of the remaining $r-1$ measurements from $K_{k}\left(x_{k}\right)$, $P_{k}\left(\mathcal{S}_{r} \mid \epsilon_{k, r}^{(i)}, x_{k}\right)$ is obtained by appropriately integrating over $\mathcal{S}_{r}$ the density $g_{k}\left(z_{k, i} \mid x_{k}\right) \prod_{j \neq i}^{r} c_{k}\left(z_{k, j} \mid x_{k}\right)$ (the product of the primary target measurement likelihood and the densities of the remaining points), resulting in (A8). That is

$$
\begin{aligned}
& P_{k}\left(\mathcal{S}_{r} \mid \epsilon_{k, r}^{(0)}, x_{k}\right)=\frac{1}{r !} \int_{\chi^{-1}\left(\mathcal{S}_{r}\right) \cap \mathcal{Z}^{r}} K_{o}^{r} \prod_{k=1}^{r} c_{k}\left(z_{k, j} \mid x_{k}\right) \\
& \times \lambda^{r}\left(d z_{k, 1} \ldots d z_{k, r}\right)
\end{aligned}
$$

$$
\begin{aligned}
P_{k}\left(\mathcal{S}_{r} \mid \epsilon_{k, r}^{(i)}, x_{k}\right)=\frac{1}{r !} \int_{\substack{\chi^{-1}\left(\mathcal{S}_{r}\right) \\
\cap \mathcal{Z}^{r} \\
\times \lambda^{r}\left(d z_{k, 1} \ldots d z_{k, r}\right)}} K_{o}^{r} g_{k}\left(z_{k, i} \mid x_{k}\right) \prod_{j \neq i}^{r} c_{k}\left(z_{k, j} \mid x_{k}\right) \\
\text { (A8) }
\end{aligned}
$$

where $\lambda^{r}$ is the $r$ th product Lebesque measure on $\mathcal{Z}^{r}$ and $\chi$ is a mapping of vectors to sets given by $\chi\left(\left[z_{1}, \ldots, z_{r}\right]^{T}\right)=\left\{z_{i}\right.$ : $i=1, \ldots, r\}$, where $K_{o}$ denotes the unit of volume on $\mathcal{Z}$, and $\mathcal{Z}^{r}$ is the $r$ th Cartesian product of $\mathcal{Z}$ with the convention $\mathcal{Z}^{0}=\{\emptyset\}$.

Hence, noting that $\chi^{-1}(\mathcal{S}) \cap \mathcal{Z}^{r}=\chi^{-1}\left(\mathcal{S}_{r}\right) \cap \mathcal{Z}^{r}$, the full expression for $P_{k}\left(\mathcal{S} \mid x_{k}\right)$ is obtained by substituting (A5), (A6) and (A7), (A8) into (A4) and using (A2) to give

$$
\begin{array}{r}
P_{k}\left(\mathcal{S} \mid x_{k}\right)=\sum_{r=0}^{\infty} \frac{1}{r !} \int_{\chi^{-1}(\mathcal{S}) \cap \mathcal{Z}^{r}} \eta_{k}\left(\left\{z_{k, 1}, \ldots, z_{k, r}\right\} \mid x_{k}\right) \\
\times \lambda^{r}\left(d z_{k, 1} \ldots d z_{k, r}\right) .
\end{array}
$$

As a check for consistency, it can be easily verified by inspection that (A9) defines a probability measure on $\mathcal{B}(\mathcal{F}(\mathcal{Z})$ ) since it is a countably additive, non-negative function which satisfies $P_{k}\left(\emptyset \mid x_{k}\right)=0$ and $P_{k}\left(\Omega \mid x_{k}\right)=1$. Finally, by comparison with the integral (6), it follows that the Radon-Nikodým derivative of $P_{k}\left(\cdot \mid x_{k}\right)$ is $\eta_{k}\left(\cdot \mid x_{k}\right)$.

\section{APPENDIX B}

The proof of Proposition 2 is given as follows.

To establish the connection for the prediction, note that since $\rho_{k-1}(\cdot)=\rho_{k \mid k-1}(\cdot)=\delta_{1}(\cdot)$, we have $v_{k-1}$ and $v_{k \mid k-1}$ are indeed the previous and predicted densities, and hence (19) for $N=1$ is exactly the single target prediction (16).

To establish the connection for the update, it is necessary to simplify (20) for $N=1$. Before proceeding, note that $\Upsilon_{k}^{0}[v, Z](1), \Upsilon_{k}^{1}[v, Z](1), \Upsilon_{k}^{1}[v, Z \backslash\{z\}](1)$ can be simplified as given in (B1), (B2), (B3), respectively, noting that by convention $P_{0}^{1}=P_{1}^{1}=1, P_{2}^{1}=0$ and $e_{0}(Z)=1, e_{1}(Z)=\sum_{z \in Z} z$.

$$
\begin{aligned}
\Upsilon_{k}^{0}[v, Z](1)= & |Z| ! p_{K, k}(|Z|) \frac{\left\langle 1-p_{D, k}, v\right\rangle}{\langle 1, v\rangle} \\
& +(|Z|-1) ! p_{K, k}(|Z|-1) \frac{1}{\langle 1, v\rangle} \\
& \times \sum_{z \in Z}\left\langle v, \psi_{k, z}\right\rangle \\
\Upsilon_{k}^{1}[v, Z](1)= & |Z| ! p_{K, k}(|Z|) \frac{1}{\langle 1, v\rangle} \\
\Upsilon_{k}^{1}[v, Z \backslash\{z\}](1)= & (|Z|-1 \mid) ! p_{K, k}(|Z-1|) \frac{1}{\langle 1, v\rangle} .
\end{aligned}
$$

Now using (B1), (B2), and (B3) in (20) with $N=1$, the special case CPHD update for $N=1$ can be simplified as shown in (B4)-(B6), shown at the top of the next page. From (B6), multiplying top and bottom by $\sum_{z \in Z} c_{k}(z)$, it follows that

$$
v_{k}(x)=\frac{\eta_{k}\left(Z_{k} \mid x\right) v_{k \mid k-1}(x)}{\int \eta_{k}\left(Z_{k} \mid x\right) v_{k \mid k-1}(x) d x} .
$$

Furthermore, since $\rho_{k \mid k-1}(\cdot)=\rho_{k}(\cdot)=\delta_{1}(\cdot)$, we have $v_{k \mid k-1}$ and $v_{k}$ are indeed the predicted and posterior densities, 


$$
\begin{aligned}
v_{k}(x) & =\frac{\left|Z_{k}\right| ! p_{K, k}\left(\left|Z_{k}\right|\right) \frac{1}{\left\langle 1, v_{k \mid k-1}\right\rangle}\left[1-p_{D, k}(x)\right] v_{k \mid k-1}(x)+\sum_{z \in Z_{k}}\left(\left|Z_{k}\right|-1\right) ! p_{K, k}\left(\left|Z_{k}-1\right|\right) \frac{1}{\left\langle 1, v_{k \mid k-1}\right\rangle} \psi_{k, z}(x) v_{k \mid k-1}(x)}{\left|Z_{k}\right| ! p_{K, k}\left(\left|Z_{k}\right|\right) \frac{\left\langle 1-p_{D, k}, v_{k}\right| k-1}{\left\langle 1, v_{k \mid k-1}\right\rangle}+\left(\left|Z_{k}\right|-1\right) ! p_{K, k}\left(\left|Z_{k}\right|-1\right) \frac{1}{\left\langle 1, v_{k \mid k-1}\right\rangle} \sum_{z \in Z_{k}}\left\langle v_{k \mid k-1}, \psi_{k, z}\right\rangle} \\
& =\frac{\left|Z_{k}\right| ! p_{K, k}\left(\left|Z_{k}\right|\right)\left[1-p_{D, k}(x)\right] v_{k \mid k-1}(x)+\sum_{z \in Z_{k}}\left(\left|Z_{k}\right|-1\right) ! p_{K, k}\left(\left|Z_{k}\right|-1\right) \frac{g_{k}(z \mid x)}{c_{k}(z)} p_{D, k}(x) v_{k \mid k-1}(x)}{\left|Z_{k}\right| ! p_{K, k}\left(\left|Z_{k}\right|\right) \int\left[1-p_{D, k}(x)\right] v_{k \mid k-1}(x) d x+\sum_{z \in Z_{k}}\left(\left|Z_{k}\right|-1\right) ! p_{K, k}\left(\left|Z_{k}\right|-1\right) \int \frac{g_{k}(z \mid x)}{c_{k}(z)} p_{D, k}(x) v_{k \mid k-1}(x) d x} \\
& =\frac{\left(\left|Z_{k}\right| ! p_{K, k}\left(\left|Z_{k}\right|\right)\left[1-p_{D, k}(x)\right]+\sum_{z \in Z_{k}}\left(\left|Z_{k}\right|-1\right) ! p_{K, k}\left(\left|Z_{k}\right|-1\right) \frac{g_{k}(z \mid x)}{c_{k}(z)} p_{D, k}(x)\right) v_{k \mid k-1}(x)}{\int\left(\left|Z_{k}\right| ! p_{K, k}\left(\left|Z_{k}\right|\right)\left[1-p_{D, k}(x)\right]+\sum_{z \in Z_{k}}\left(\left|Z_{k}\right|-1\right) ! p_{K, k}\left(\left|Z_{k}\right|-1\right) \frac{g_{k}(z \mid x)}{c_{k}(z)} p_{D, k}(x)\right) v_{k \mid k-1}(x) d x}
\end{aligned}
$$

hence by (B7) the special case CPHD update (20) for $N=1$ is exactly the RFS single-target Bayes' update (17).

\section{APPENDIX C}

Propositions 3 and 4 are established using the following standard results for Gaussian functions; for further details, see, e.g., [18] and [35].

Lemma 1: Given $F, d, Q, m, P$ of matching dimensions, where $Q$ and $P$ are positive definite, $\int \mathcal{N}(x ; F \zeta+$ $d, Q) \mathcal{N}(\zeta ; m, P) d \zeta=\mathcal{N}\left(x ; F m+d, Q+F P F^{T}\right)$

Lemma 2: Given $H, b, R, m, P$ of matching dimensions, where $R$ and $P$ are positive definite, $\mathcal{N}(z ; H x+$ $b, R) \mathcal{N}(x ; m, P)=q(z) \mathcal{N}(x ; \tilde{m}, \tilde{P})$, where $q(z)=$ $\mathcal{N}\left(z ; H m+b, R+H P H^{T}\right), \tilde{m}=m+K(z-H m-b)$, $\tilde{P}=(I-K H) P, K=P H^{T}\left(R+H P H^{T}\right)^{-1}$.

Proposition 3 is the prediction step of the Gaussian sum filter [30]. For completeness, it is obtained by substituting (29) and (35) into (16) and replacing integrals of products of Gaussians as given in Lemma 1. Proposition 4 is established as follows. The closed-form expression for $\left(\Xi_{z} \phi\right)(x)$ given in (58) is obtained by multiplying (57) and (42) and replacing products of Gaussians with single Gaussians as given in Lemma 2. Proposition 4 is then obtained by substituting (49) into the numerator of (17) and using the operators $G_{k, z}$ and $C_{k, z}$ to convert products of Gaussian mixtures into a single mixture as appropriate. The normalizing constant follows straightforwardly from substituting (53) into (17).

\section{REFERENCES}

[1] D. Reid, "An algorithm for tracking multiple targets," IEEE Trans. Autom. Control, vol. AC-24, no. 6, pp. 843-854, Dec. 1979.

[2] S. Blackman, Multiple Target Tracking With Radar Applications. Norwood, MA: Artech House, 1986.

[3] Y. Bar-Shalom and T. E. Fortmann, Tracking and Data Association. San Diego, CA: Academic, 1988.

[4] D. Salmond, "Mixture reduction algorithms for target tracking in clutter," in Proc. SPIE Signal and Data Processing of Small Targets, 1990, vol. 1305, pp. 434-445.

[5] D. Musicki, R. Evans, and S. Stankovic, "Integrated probabilistic data association," IEEE Trans. Autom. Control, vol. AC-39, no. 6, pp. 1237-1241, Jun. 1994.

[6] S. Mori, C. Y. Chong, E. Tse, and R. Wishner, "Tracking and classifying multiple targets without a priori identification," IEEE Trans. Autom. Control, vol. AC-31, no. 5, pp. 401-409, May 1986.
[7] R. Washburn, "A random point process approach to multi-object tracking," in Proc. American Control Conf., 1987, vol. 3, pp. $1846-1852$

[8] N. Portenko, H. Salehi, and A. Skorokhod, "On optimal filtering of multitarget systems based on point process," Rand. Oper. Stoch. Eq., vol. 5, no. 1, pp. 1-34, 1997.

[9] I. Goodman, R. Mahler, and H. Nguyen, Mathematics of Data Fusion. Norwell, MA: Kluwer Academic, 1997.

[10] B.-N. Vo, S. Singh, and A. Doucet, "Sequential Monte Carlo methods for multi-target filtering with random finite sets," IEEE Trans. Aerosp. Electron. Syst., vol. 41, no. 4, pp. 1224-1245, Oct. 2005.

[11] R. Mahler, Statistical Multisource-Multitarget Information Fusion. Norwood, MA: Artech House, 2007.

[12] R. Mahler, "Multi-target Bayes filtering via first-order multi-target moments," IEEE Trans. Aerosp. Electron. Syst., vol. 39, no. 4, pp. 1152-1178, Oct. 2003.

[13] B.-N. Vo and W.-K. Ma, "The Gaussian mixture probability hypothesis density filter," IEEE Trans. Signal Process., vol. 54, no. 11, pp. 4091-4104, Nov. 2006

[14] R. Mahler, "PHD filters of higher order in target number," IEEE Trans. Aerosp. Electron. Syst., vol. 43, no. 3, pp. 1523-1543, Oct. 2007.

[15] B.-T. Vo, B.-N. Vo, and A. Cantoni, "Analytic implementations of the cardinalized probability hypothesis density filter," IEEE Trans. Signal Process., vol. 55, no. 7, pp. 3553-3567, Jul. 2007.

[16] S. Challa, B.-N. Vo, and X. Wang, "Bayesian approaches to track existence-IPDA and random sets," presented at the 5th Int. Conf. Information Fusion, Annapolis, MD, May 2002.

[17] B.-T. Vo, B.-N. Vo, and A. Cantoni, "A Bayesian approach to target tracking with finite-set-valued observations," presented at the 22nd Int. Symp. Intelligent Control, Singapore, Oct. 2007.

[18] B. Ristic, S. Arulampalam, and N. Gordon, Beyond the Kalman Filter: Particle Filters for Tracking Applications. Norwood, MA: Artec House, 2004.

[19] A. Doucet, N. de Freitas, and N. Gordon, Sequential Monte Carlo Methods in Practice. New York: Springer-Verlag, 2001.

[20] D. Daley and D. Vere-Jones, An Introduction to the Theory of Point Processes. New York: Springer-Verlag, 1988.

[21] D. Stoyan, D. Kendall, and J. Mecke, Stochastic Geometry and its Applications. New York: Wiley, 1995.

[22] J. Moller and R. Waagepetersen, Statistical Inference and Simulation for Spatial Point Processes. London, U.K.: Chapman \& Hall/CRC, 2004.

[23] M. N. van Lieshout, Markov Point Processes and Their Applications. London, U.K.: Imperial College Press, 2000.

[24] M. Ulmke, O. Erdinc, and P. Willett, "Gaussian mixture cardinalized PHD filter for ground moving target tracking," presented at the 10th Int. Conf. Information Fusion, Quebec, Canada, Jul. 2007.

[25] S. Arulampalam, S. Maskell, N. J. Gordon, and T. Clapp, "A tutorial on particle filters for on-line non-linear/non-Gaussian Bayesian tracking," IEEE Trans. Signal Process., vol. 50, no. 2, pp. 174-188, Feb. 2002.

[26] D. Crisan and A. Doucet, "A survey of convergence results on particle filtering methods for practitioners," IEEE Trans. Signal Process., vol. 50, no. 3, pp. 736-746, Mar. 2002. 
[27] P. D. Moral, Feynman-Kac Formulae:Genealogical and Interacting Particle Systems with Applications. New York: Springer-Verlag, 2004.

[28] R. Gilks and C. Berzuini, "Following a moving target: Monte Carlo inference for dynamic Bayesian models," J. Roy. Stat. Soc., vol. 63, no. 1, pp. 127-146, 2001.

[29] X.-R. Li and V. Jilkov, "Survey of maneuvering target tracking," IEEE Trans. Aerosp. Electron. Syst., vol. 39, no. 4, pp. 1333-1364, 2003.

[30] H. W. Sorenson and D. L. Alspach, "Recursive Bayesian estimation using Gaussian sums," Automatica, vol. 7, pp. 465-479, 1971.

[31] K. Murphy and S. Russell, "Rao-Blackwellised particle filtering for dynamic Bayesian networks," in Sequential Monte Carlo Methods in Practice, A. Doucet, N. Freitas, and N. Gordon, Eds. New York: Springer-Verlag, 2001, pp. 499-515.

[32] A. H. Jazwinski, Stochastic Processes and Filtering Theory. New York: Academic, 1970

[33] B. D. Anderson and J. B. Moore, Optimal Filtering. Englewood Cliffs, NJ: Prentice-Hall, 1979

[34] S. J. Julier and J. K. Uhlmann, "Unscented filtering and nonlinear estimation," Proc. IEEE, vol. 92, no. 3, pp. 401-422, 2004.

[35] Y. C. Ho and R. C. K. Lee, "A Bayesian approach to problems in stochastic estimation and control," IEEE Trans. Autom. Control, vol. AC-9, no. 4, pp. 333-339, Oct. 1964.

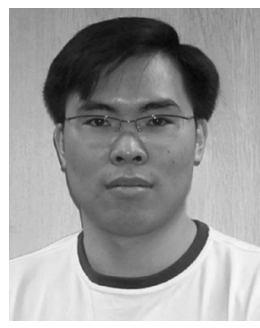

Ba-Tuong Vo was born in Perth, Australia, 1982. He received the B.Sc. degree in mathematics and the B.E. degree in electrical engineering (with first-class honors) from the University of Western Australia, Crawley, in 2004. He is currently working towards the Ph.D. degree in electrical engineering with the Western Australian Telecommunications Research Institute at the University of Western Australia.

His research interests include point processes, filtering and estimation, and multi-object tracking.

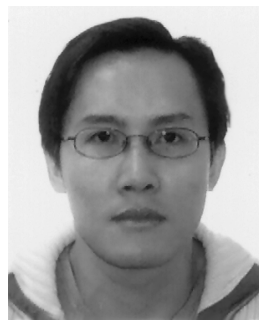

Ba-Ngu Vo was born in Saigon, Vietnam, 1970. He received the Bachelor's degrees jointly in science and electrical engineering (with first-class honors) at the University of Western Australia, Crawley, in 1994, and the $\mathrm{Ph} . \mathrm{D}$. degree in electrical engineering from Curtin University of Technology, Perth, WA, Australia, in 1997.

He has held various research positions in Australia and overseas before joining the Department of Electrical and Electronic Engineering at the University of Melbourne, Australia, in 2000, where he is currently an Associate Professor. His research interests include optimization, signal processing, and stochastic geometry.

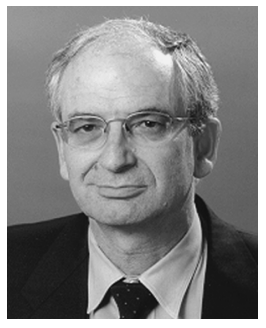

Antonio Cantoni (M'74-SM'83-F'98) was born in Soliera, Italy, on October 30, 1946. He received the B.E. (first-class honors) and Ph.D. degrees in electrical engineering from the University of Western Australia, WA, Australia, in 1968 and 1972, respectively.

$\mathrm{He}$ was a Lecturer in computer science at the Australian National University, Canberra, Australia, in 1972. He joined the Department of Electrical and Electronic Engineering at the University of Newcastle, NSW, Australia, in 1973, where he held the Chair of Computer Engineering until 1986. In 1987, he joined QPSX Communications Ltd., Perth, WA, Australia, as Director of the Digital and Computer Systems Design Section for the development of the DQDB metropolitan area network. From 1987 to 1990, he was also a Visiting Professor in the Department of Electrical and Electronic Engineering at the University of Western Australia, Nedlands, WA, Australia. From 1992 to 1997, he was the Director of the Western Australian Telecommunications Research Institute and Professor of Telecommunications at Curtin University of Technology, Perth, WA, Australia. During this period, he was also the Director of the Cooperative Research Centre for BroadBand Telecommunications and Networking. From 1997 to 2000, he was Chief Technology Officer of Atmosphere Networks an optical networks startup that he co-founded. Since 2000, he has been Research Director of the Western Australian Telecommunications Research Institute and Professor of Telecommunications at the University of Western Australia. He is currently a consultant to Sensear Pty Ltd and Ispire Networks Pty. Ltd. startups that he co-founded. His research interests include adaptive signal processing, electronic system design, phase-locked loops, and networking.

Dr. Cantoni is a Fellow of the Australian Academy of Technological Sciences and Engineering. He has been an Associate Editor of the IEEE TRANSACTIONS ON SIGNAL PROCESSING. 